\title{
Performance Evaluation of a Novel and Effective Water-Soluble Aldehydes as Corrosion Inhibitor for Carbon Steel in Aggressive Hydrochloric Medium
}

\author{
Yun Wang ${ }^{1}$, Tiantian Wang ${ }^{2}$, Bei Wang ${ }^{1}$, Wei Chang ${ }^{3}$, Jiangli Cao ${ }^{1}$, Lihua $\mathrm{Hu}^{3}$, Minxu $\mathrm{Lu}^{1}$ and \\ Lei Zhang ${ }^{1, *}$ \\ ${ }^{1}$ University of Science and Technology Beijing, Beijing, 100083, China \\ ${ }^{2}$ Wenzhou Hongfeng Electrical Alloy Co., Ltd., Wenzhou, 325603, China \\ ${ }^{3}$ CNOOC Research Institute, Beijing, 100028, China \\ ${ }^{*}$ Corresponding Author: Lei Zhang. Email: zhanglei@ustb.edu.cn \\ Received: 24 December 2020 Accepted: 11 March 2021
}

\begin{abstract}
A novel and effective water-soluble aldehydes ( $\beta$-HA) as corrosion inhibitor was synthesized for N80 steel corrosion in $15 \% \mathrm{HCl}$ solution, and the corrosion inhibition performance was evaluated by using weight loss, electrochemical measurements, scanning electron microscope (SEM), quantum chemical calculation and molecular dynamics simulation (MDS). The results show that synthesized $\beta$-HA showed excellent corrosion performance compared with $\mathrm{MHB}$ and PE for carbon steel in $15 \% \mathrm{HCl}$ solution compared with $\mathrm{MHB}$ and $\mathrm{PE}$, and the inhibition efficiency increased with increasing concentration of the inhibitor. The inhibition efficiency of $\beta$-HA at $8 \mathrm{mmol} / \mathrm{L}$ reached the maximum value $94.08 \%$. The inhibitor acted as mixed-type inhibitor via blocking both the anodic and cathodic reaction. The adsorption of inhibitors on N80 steel surface obeyed Langmuir adsorption isotherm, and the process contained chemisorption and physisorption. The $\Delta G_{\text {ads }}$ of $\beta$-HA was $-28.81 \mathrm{~kJ} \cdot \mathrm{mol}^{-1}$ under the standard atmospheric pressure. Moreover, the theoretical calculation parameters revealed stronger combination and higher interaction energy for inhibitor $\beta$-HA comparing with MHB and PE, further demonstrating the correlation between the theoretical and experimental results.
\end{abstract}

\section{KEYWORDS}

Corrosion; inhibitors; n80 steel; electrochemical; adsorption; molecular dynamics simulation

\section{Introduction}

The corrosion of carbon steel, a common material used in petroleum and biomedicine industries, has become increasingly prominent [1-8]. In industrial production, sulfuric acid and hydrochloric acid are the most widely used pickling agents [9]. Due to the strong corrosivity of acid, acid can remove rust and dirt, but also have a strong corrosive effect on the metal part of the substrate, and serious corrosion of the equipment will bring huge economic losses and harm. In order to solve the problem of metal material loss caused by pickling every year in China, corrosion scientists have developed a variety of solutions, such as surface coating, bond coating, corrosion inhibitor, etc. [10]. Numerous physical, chemical and mechanical methods, the addition of corrosion inhibitor is most effective and economical approach to prevent corrosion in the corrosive media [11-13]. Some effective inhibitors have been developed such as 
imidazoline and pyran derivatives $[14,15]$. However, most of the derivatives are toxic, and it can cause dizziness, insomnia, or even liver damage to human. In view of the shortcomings of traditional corrosion inhibitors, it is necessary to develop green and environmental-friendly corrosion inhibitors, especially for the food, biological and pharmaceutical industries.

Plant-type corrosion inhibitor is used in industrial production to inhibit the corrosion of metal materials in corrosive environment, which is a new type of natural green corrosion inhibitor. To date, some green inhibitors have been derived from natural products or synthesized [16-18]. Ma et al. prepared a supramolecular complex (CDCA) based on $\beta$-cyclodextrin $(\beta-\mathrm{CyD})$ and trans-cinnamaldehyde (MHB) through organic solvent extraction. The inhibition efficiency for mild steel in $\mathrm{HCl}$ solution could reach 93.8\% in the presence of $12 \mathrm{mM} \mathrm{CDCA}$, and both the cathodic and anodic process could be depressed in the presence of CDCA, which was categorized as the mix-type inhibitior [19]. Su et al. investigated the corrosion inhibitive performance of a new schiff base N, N'-dicinnamylidene-o-phenylenediamine as acid inhibitor by the condensation of ophenylenediamine and cinnamaldehyde. The results showed that DCPD could effectively inhibit the corrosion to carbon steel in $10 \% \mathrm{HCl}$ solution [20]. El-Etre [21] find vanillin can achieve $99 \%$ surface coverage on aluminum surface by the adsorption through the active contained in its structure [21]. 3-Methoxy-4-hydroxybenzaldehyde can also be used as the inhibitors in food system, e.g., the oxidation of dihydrorhodamine 123 to fluorescent rhodamine 123 was inhibited by vanillin [22]. Furthermore, (E)-3-phenylprop-2-enal can also be used as a good inhibition against steel corrosion by formation of a continuously thickening polymer film [23]. Gupta et al. manifested that L-lysine and aromatic aldehydes, as green corrosion inhibitors for mild steel, exhibited a maximum inhibition efficiency of $95.6 \%$ at $400 \mathrm{mg} \cdot \mathrm{L}^{-1}$ concentration, and adsorption of the SBs (Schiff's bases) on mild steel surface obeys the Langmuir adsorption isotherms [24]. These corrosion inhibitors are derived or synthesized from natural products. They are able to become excellent corrosion inhibitors, while reducing the potentially negative impact to nature and human being. In addition to furfural, 3-Methoxy-4hydroxybenzaldehyde (MHB) and (E)-3-phenylprop-2-enal (PE) also have the characteristics of high efficiency and environmental-friendliness. PE is recognized as a safe and non-toxic substance by the U.S. Food and Drug Administration. It is commonly used as food and spice additives. MHB, as the principal raw material for aroma modification, is widely used in food, soap, tobacco, drugs and other light industries. Technically, the function of corrosion inhibition is mainly achieved through the formation a polymeric film on the surface. PE and MHB contain many functional groups, such as aromatic groups, carbonyl groups, hydroxyl groups, and methoxy groups, which can easily form a film through adsorption. Therefore, it is hypothesized that PE and MHB can be used as inhibitors due to abundant adsorption groups.

In this paper, we developed a novel corrosion inhibitor $\beta$-HA by catalyst-free green method, and its structure was characterized by Fourier transform infrared (FT-IR) and 1H NMR spectra. The corrosion resistance of $\beta$-HA was studied by scanning electron microcopy (SEM), weight-loss method and electrochemical methods to verify the effectiveness for protection and reveal the mechanisms of corrosion prevention. Quantum chemical calculation and molecular dynamics simulation were utilized to study the electronic properties and the interaction between inhibitor molecule and metal surface.

\section{Materials and Methods}

\subsection{Preparation of Electrode and Solution}

N80 steel was used for the corrosion test. Its chemical composition is shown in Tab. 1. All specimens $\left(1 \times 1 \mathrm{~cm}^{2}\right)$ were polished using 360 1200 grit sandpapers and then cleaned with deionized water and ethanol, respectively. Hydrochloric acid (36.5\%, Beijing Chemical Plant), acetone analytical reagent ( $\geq 99.5 \%$, Tianjin Bohua Chemical Reagent Co., Ltd., China), absolute ethyl alcohol ( $\geq 99.7 \%$, Lizhi Chemical Engineering), 3-Methoxy-4-hydroxybenzaldehyde (MHB) (99\%, Sigma-Aldrich), (E)-3phenylprop-2-enal (PE) (93\%, Sigma-Aldrich) were purchased, all of the reagents were analytical grades. The test corrosion solution is $15 \% \mathrm{HCl}$ solution, which is made of $36.5 \%$ concentrated $\mathrm{HCl}$ (AR grade) diluted with distilled water. 
Table 1: Chemical composition of N80 steel in the experimental (wt\%)

\begin{tabular}{|c|c|c|c|c|c|c|c|c|c|c|}
\hline $\mathrm{Fe}$ & $\mathrm{C}$ & $\mathrm{Si}$ & $\mathrm{Mn}$ & $\mathrm{P}$ & $\mathrm{S}$ & $\mathrm{Cr}$ & Mo & $\mathrm{Ni}$ & $\mathrm{Ti}$ & $\mathrm{Cu}$ \\
\hline 97.468 & 0.42 & 0.24 & 1.55 & 0.012 & 0.004 & 0.051 & 0.18 & 0.005 & 0.01 & 0.06 \\
\hline
\end{tabular}

\subsection{Preparation of 3-methoxy-4-hydroxybenzaldehyde (MHB) and (e)-3-phenylprop-2-enal (PE)}

In order to analyze the corrosion inhibition performance of MHB and PE for $\mathrm{N} 80$ steel corrosion in 15\% $\mathrm{HCl}$ solution, the concentrations of inhibitor MHB and PE were prepared as $0,2,4,6$ and $8 \mathrm{mmol} \cdot \mathrm{L}^{-1}$.

\subsection{Preparation of $\beta-h A$}

The preparation of $\beta$-HA is shown in Fig. 1 . When preparing the $\beta$-HA corrosion inhibitor, 2 mmol of MHB powder was first weighed and dissolved in $50 \mathrm{ml}$ deionized water, followed by $4 \mathrm{mmol}$ PE ( $4 \mathrm{ml}$, $1 \mathrm{~mol} \cdot \mathrm{L}^{-1}$ ) solution and $5 \mathrm{ml}$ dilute $\mathrm{NaOH}$ with stirring. Finally, the solution was transferred to a Teflonlined stainless-steel autoclave and then heated at $120^{\circ} \mathrm{C}$ for $4 \mathrm{~h}$. After cooling to room temperature, the reaction solution was centrifuged for $15 \mathrm{~min}$ [25].
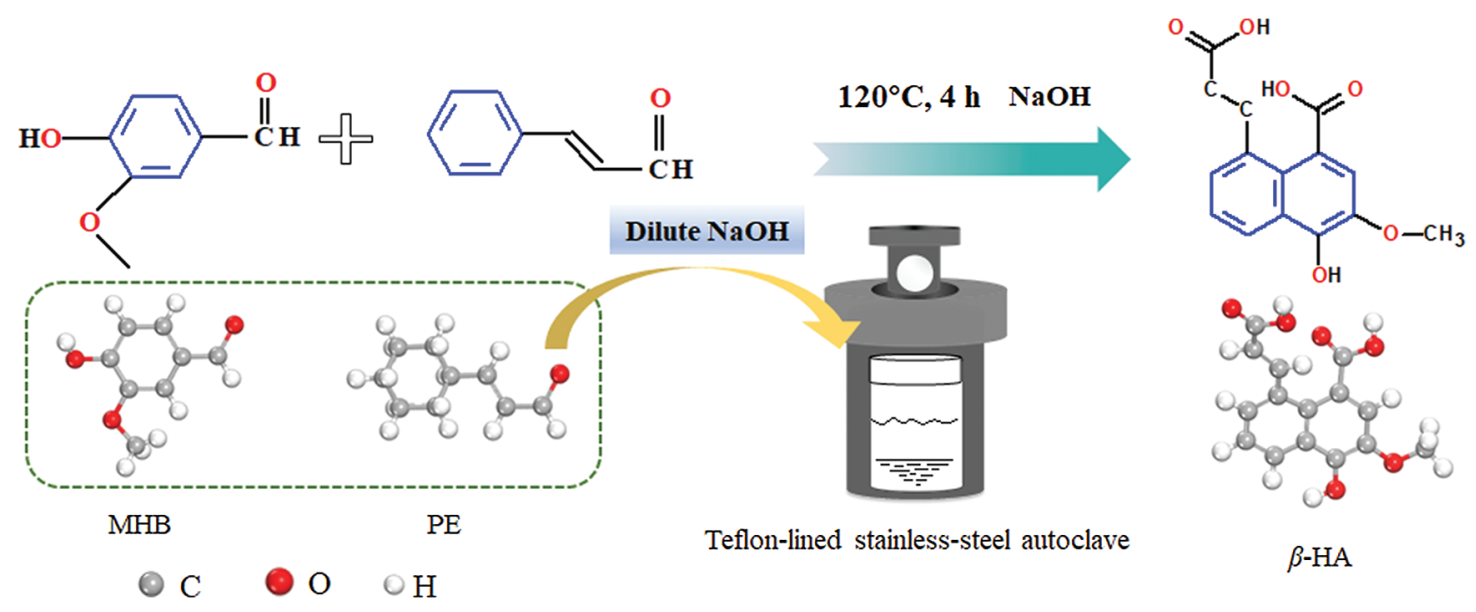

Figure 1: Synthesis procedure of inhibitor $\beta$-HA

\subsection{Preparation of $\beta$-hA Solution}

To compare the performance of corrosion inhibitor $\beta$-HA, the solution with the same concentration as MHB and PE was prepared.

\subsection{Structure Characterization}

Structure of $\beta$-HA was characterized using Thermo Fisher Scientific Nicolet 6700 FTIR and 1H NMR spectra was recorded in $\mathrm{CDCl}_{3}$ by a Bruker digital FT-NMR spectrometer (Bruker BioSpin $\mathrm{GmbH}$ ).

\subsection{Weight Loss Experiments}

All samples were exposed to $15 \% \mathrm{HCl}$ solution in the absence and presence of $\beta$-HA for $12 \mathrm{~h}$ at $303 \mathrm{~K}$ and different immersion time. Prior to each test, the samples were weighed three times using a digital weighing balance of precision $\pm 0.01 \mathrm{mg}$. After the experiment, the samples were taken out and washed thoroughly in running water and distilled water, rinsed in acetone, and finally dried in a stream of warm 
air. The dried specimens were reweighed to calculate the corrosion rate (v), which was calculated by following Eq. (1) [26]:

$v=\frac{K \times \Delta W}{A \times t \times \rho}$

where $K$ is the constant $\left(8.76 \times 10^{4}\right), \Delta W$ is the mass loss $(\mathrm{g}), A$ is the surface area of the specimen $\left(\mathrm{cm}^{2}\right), t$ is the immersion time (h) and $\rho$ is the density of the steel sample $\left(\mathrm{g} / \mathrm{cm}^{3}\right)$.

\subsection{Electrochemical Measurements}

A conventional three-electrode system (working electrode: N80 steel with area of $0.85 \mathrm{~cm}^{2}$, counter electrode: $\mathrm{Pt}$, reference electrode: a saturated calomel electrode) was used in all electrochemical measurements by CS350 electrochemical workstation. The sweeping rate during polarization measurements was $0.5 \mathrm{mV} / \mathrm{s}$, and the potential was changed from -0.5 to $+0.5 \mathrm{~V}$ ( $v s$. SCE). Electrochemical impedance spectroscopy (EIS) was performed at the open-circuit potential in the frequency range from 0.01 to $100 \mathrm{kHz}$ with an excitation signal of $10 \mathrm{mV}$. The electrode potential was allowed to stabilize for $30 \mathrm{~min}$ before starting the measurements. Each experiment was repeated three times to ensure repeatability. The experimental schematic diagram of electrochemical device is shown in Fig. 2.

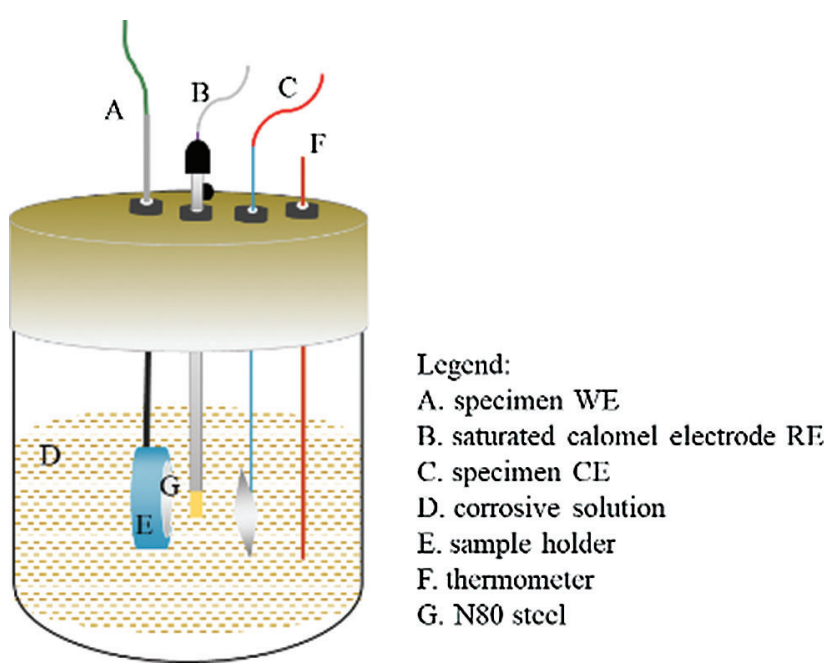

Figure 2: Schematic diagram of the electrochemical device

\subsection{Surface Characterization}

The corrosion morphology of specimen was observed by scanning electron microscope (SEM, S4800, Japan) and the electron gun acceleration voltage was $10 \mathrm{kV}$. X-ray photoelectron spectroscopy (XPS) was obtained using a Shimadzu-Kratos AXIS Ultra DLD with $\mathrm{Al} \mathrm{K} \alpha$ as excitation source, and the binding energy was calibrated by $\pm 0.1 \mathrm{eV} \mathrm{C1} \mathrm{s}$ peaking at $284.6 \mathrm{eV}$ [27]. The three-dimensional morphology of steel surface was observed using Olympus.

\subsection{Computational Details}

All quantum chemical calculations were performed at the BLYP-D3BJ/def2-SVP level using Density Functional Theory (DFT) using the GGA functional and the DNP basis set by using Materials Studio8.0 package with Density Functional Theory (DFT) [28,29]. This basis set can guarantee the 
precise geometric and electronic properties for organic molecules. Initial structural optimization of the three inhibitors was carried out with DFT-D correction at the convergence thresholds of energy change of $1.0 \times 10^{-5} \mathrm{Ha}$, the maximum force of $0.002 \mathrm{Ha} / \AA$ and maximum displacement of $5.0 \times 10^{-3} \AA$. In addition, the global orbital cutoff and the self-consistent field tolerance are $4.6 \AA$ and $1.0 \times 10^{-6} \mathrm{Ha}$, respectively. Theoretical parameters such as the energy of the highest occupied orbital $\left(E_{\mathrm{HOMO}}\right)$, energy of lowest unoccupied molecular orbitals $\left(E_{\mathrm{LUMO}}\right)$, chemical potential $(\chi)$, energy gap $(\Delta E)$, global hardness $(\eta)$, global chemical softness $(\sigma)$, global electrophilicity index $(\omega)$ and Nucleophilicity $(\varepsilon)$ were calculated as follows:

$\Delta E=E_{\mathrm{LUMO}}-E_{\mathrm{HOMO}}$

$\chi=-\frac{E_{\mathrm{HOMO}}+E_{\mathrm{LUMO}}}{2}$

$\eta=-\frac{E_{\mathrm{HOMO}}-E_{\mathrm{LUMO}}}{2}$

$\sigma=-\frac{2}{E_{\mathrm{HOMO}}+E_{\mathrm{LUMO}}}$

$\omega=\frac{\mu^{2}}{2 \eta}=\frac{\chi^{2}}{2 \eta}$

$\epsilon=\frac{1}{\omega}$

For molecular dynamics (MD) simulation, Fe (110) was selected due to it has the good stability among all the crystal planes of iron. The dimension of constructed simulation cells for $\mathrm{Fe}$ (110) with 5-layer atom was as $24.82 \times 24.82 \times 48.80 \AA^{3}$. A medium slab containing $85 \mathrm{H}_{2} \mathrm{O}$ molecules, $20 \mathrm{H}_{3} \mathrm{O}^{+}, 10 \mathrm{Cl}^{-}$and 1 inhibitor molecule was built using Amorphous cell module. When the layer was built by means of Layer builder module, a large vacuum space of $20 \AA$ was placed in the $Z$ direction perpendicular to the $\mathrm{Fe}(110)$ surface and the corrosion medium layer in order to limit the interaction between inhibitor molecules and the side of Fe substrate. The geometry-optimized structures of MHB, PE and $\beta$-HA molecule were obtained using Forcite module with the force field COMPASS, which is successful in the previous study of polymer-metal interaction [30]. The built layer is minimized by using smart minimizer. Later, $500 \mathrm{ps}$ with the time step $0.1 \mathrm{fs}$ of MD simulation for the minimum simulation unit at $303 \mathrm{~K}$ was carried out to make the simulation unit reach an equilibrium state, followed by simulating in NVE ensemble for 1000 ps. The interaction between van der Waals (vdW) and electrostatic non-bonding was calculated by atomic cut-off method and electronic Wald method, respectively [31]. The operating temperature was monitored by the Andersen thermostat [32]. During calculating, all $\mathrm{Fe}$ atoms and molecules in the medium layer were frozen.

$\Delta E_{\text {binding }}$ is used to quantitatively evaluate the bonding strength of organic molecules on metals, and calculated as following:

$E_{\text {binding }}=E_{\text {total }}-E_{\text {molecule }}+E_{\text {surface }}$

where, $E_{\text {binding }}$ is the binding energy, $E_{\text {molecule }}$ is the potential energy of inhibitor molecule; $E_{\text {surface }}$ is the potential energy of $\mathrm{Fe}(110)$ surface in absence of inhibitor; $E_{\text {total }}$ is the total potential energy of the simulated layer. 


\section{Results and Discussion}

\subsection{Characterization of $\beta$ - $h A$}

FTIR, ${ }^{1} \mathrm{H}$ NMR and ${ }^{13} \mathrm{C}$ NMR spectra characterized the chemical structures of $\beta$-HA is shown in Fig. 3. For FTIR spectrum (Fig. 3a), some functional groups were presented in $\beta$-HA, including C-O-C stretching vibration $\left(1021 \mathrm{~cm}^{-1}\right)$, -C-O stretching vibration $\left(1114.0 \mathrm{~cm}^{-1}\right)$, -OH stretching vibration $\left(1402.2 \mathrm{~cm}^{-1}\right)$, $-\mathrm{C}=\mathrm{C}$ stretching vibration $\left(1315.9 \mathrm{~cm}^{-1}\right)$ in benzene ring structure, $-\mathrm{C}=\mathrm{O}$ stretching vibration $\left(1632.1 \mathrm{~cm}^{-1}\right)$, $-\mathrm{C}-\mathrm{H}$ stretching vibration $\left(2853.3 \mathrm{~cm}^{-1}\right)$ and $-\mathrm{O}-\mathrm{H}$ stretching vibration $\left(3339.1 \mathrm{~cm}^{-1}\right)$ [33]. Based on FTIR spectra results, the formation of carboxyl - $\mathrm{COOH}$ indicated that MHB and PE has a certain aldehyde-aldehyde condensation reaction.

It can be seen from Fig. $3 \mathrm{~b}$ that the absorption peak of $\mathrm{CDCl}_{3}$ is at $7.46 \mathrm{ppm}$. In addition, $\delta \mathrm{H}_{\mathrm{ppm}}=2.39$ $(-\mathrm{OH}), \delta \mathrm{H}_{\mathrm{ppm}}=2.61\left(-\mathrm{CH}_{2}\right), \delta \mathrm{H}_{\mathrm{ppm}}=3.99\left(-\mathrm{CH}_{3}\right), \delta \mathrm{H}_{\mathrm{ppm}}=7.44$ (benzene ring), and $\delta \mathrm{H}_{\mathrm{ppm}}=8.66$ (phenol hydroxyl) [34]. ${ }^{13} \mathrm{C}$ NMR (Fig. 3c) showed that the compound exists 16 carbon signals, among which $\delta \mathrm{C}_{\mathrm{ppm}}$ 168.99 , 177.8 was presumed to be carboxyl carbon signal, $\delta \mathrm{C}_{\mathrm{ppm}} 145.56,146.08,128.58$, 127.36 and 126.61 were conjugated double bond carbon signal, $\delta \mathrm{C}_{\mathrm{ppm}} 56.36$ was phenoxymethyl carbon signal, $\delta \mathrm{C}_{\mathrm{ppm}} 35.05$ and 29.26 were methylene carbon signal. Combined with the data of ${ }^{1} \mathrm{H} \mathrm{NMR}$ and ${ }^{13} \mathrm{C}$ NMR spectra, it is speculated that the compound has the carboxyl group.

\subsection{Weight Loss Measurement}

The corrosion rates of $\mathrm{N} 80$ steel exposed to $15 \% \mathrm{HCl}$ solution in the absence and presence of inhibitor for $12 \mathrm{~h}$ at $303 \mathrm{~K}$ and immersion time were obtained by weight loss in Fig. 4. The inhibition efficiencies ( $\eta$ \%) can be calculated by using the Eq. (9):

$\eta=\frac{v_{\text {corr }}^{0}-v_{\text {corr }}^{\prime}}{v_{\text {corr }}^{0}} \times 100$

where, $v_{\text {corr }}^{0}$ and $v_{\text {corr }}^{\prime}$ are rate of corrosion with and without inhibitor.

After being soaked into the solution with corrosion inhibitors MHB, PE and $\beta$-HA for $12 \mathrm{~h}$, the corrosion rates of N80 steel decreased (Fig. 4a).

In addition, the decrement gradually decreased with the increase of inhibitor concentration. The presence of $8 \mathrm{mmol} \cdot \mathrm{L}^{-1}$ inhibitor in the acid solution was sufficient to reduce the corrosion rate of the $\mathrm{N} 80$ steel from $21.56 \mathrm{~mm} / \mathrm{a}$ to $3.03 \mathrm{~mm} / \mathrm{a}, 2.85 \mathrm{~mm} / \mathrm{a}, 1.8 \mathrm{~mm} / \mathrm{a}$, respectively. The maximum inhibition efficiency for $\mathrm{MHB}, \mathrm{PE}$ and $\beta$-HA were $85.95,86.78$ and $91.65 \%$ at $8 \mathrm{mmol} \cdot \mathrm{L}^{-1}$ concentration (Tab. 2), respectively. For immersion time (Fig. 4b), the corrosion rate of steel in $15 \% \mathrm{HCl}$ solution showed an upward trend overall with the increase of immersion time, while an opposite trend was observed at $8 \mathrm{mmol} \cdot \mathrm{L}^{-1}$ inhibitor $\mathrm{MHB}, \mathrm{PE}$ and $\beta$-HA, respectively. Furthermore, the inhibition effect of $\beta$-HA inhibitor was verified.

\subsection{Open Circuit Potential}

Fig. 5 shows the OCP curves of $\mathrm{N} 80$ steel immersed in $15 \% \mathrm{HCl}$ solution at $303 \mathrm{~K}$. It can be seen from Fig. 5 that after immersing for $1800 \mathrm{~s}$, the OCP floating range is within $10 \mathrm{mV}$, and the system reaches a stable state. In addition, it is noticed that the initial value of OCP with inhibitor is higher than that without inhibitor, which may be related to the adsorption of inhibitor molecules on the surface of N80 steel [35]. 
JRM, 2022, vol.10, no.2

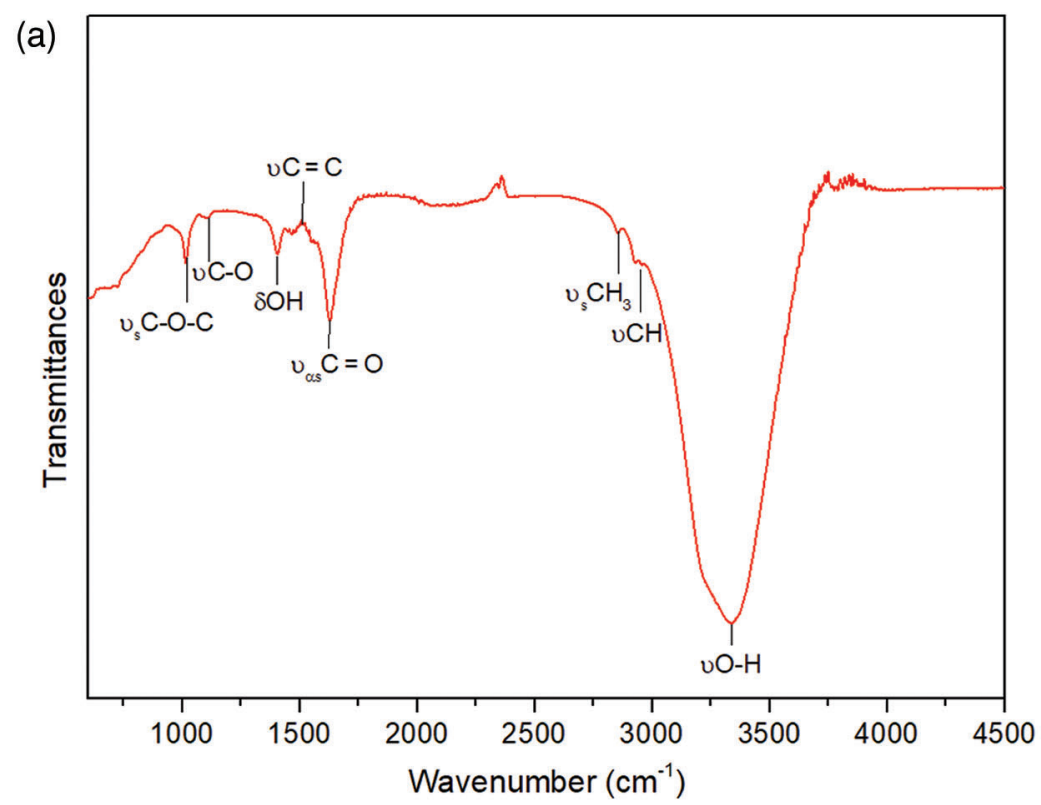

(b)

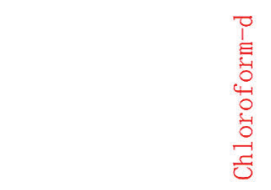

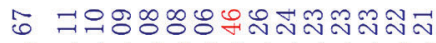

i

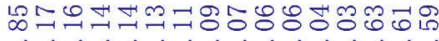

कं<smiles></smiles>

21

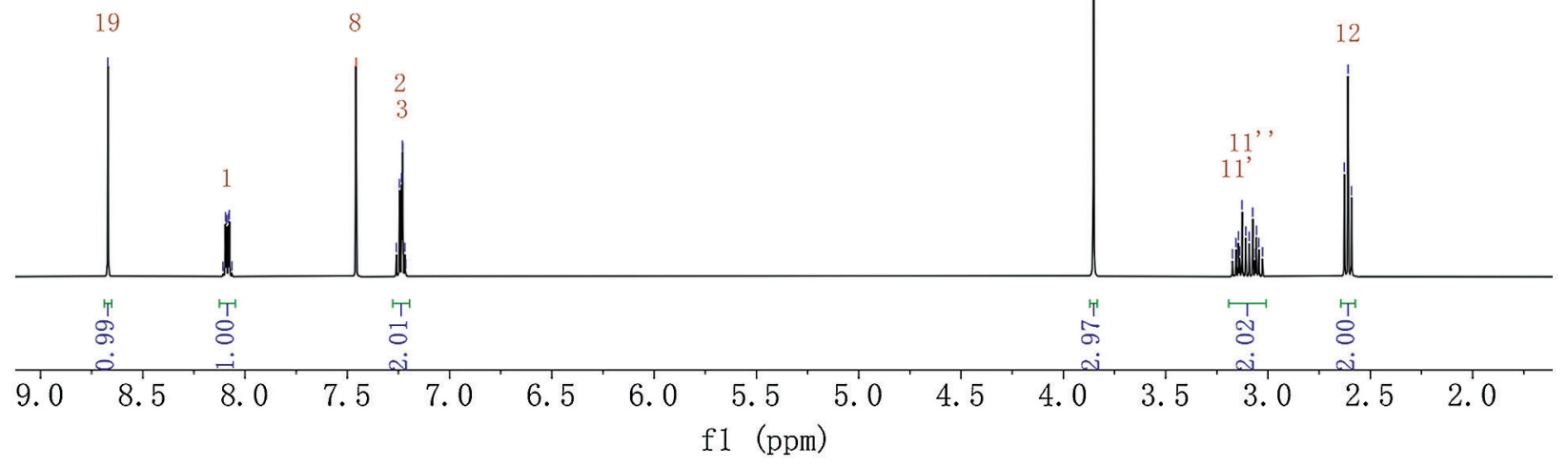

Figure 3: (Continued) 


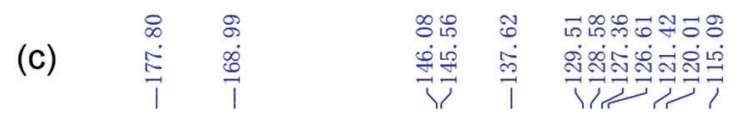
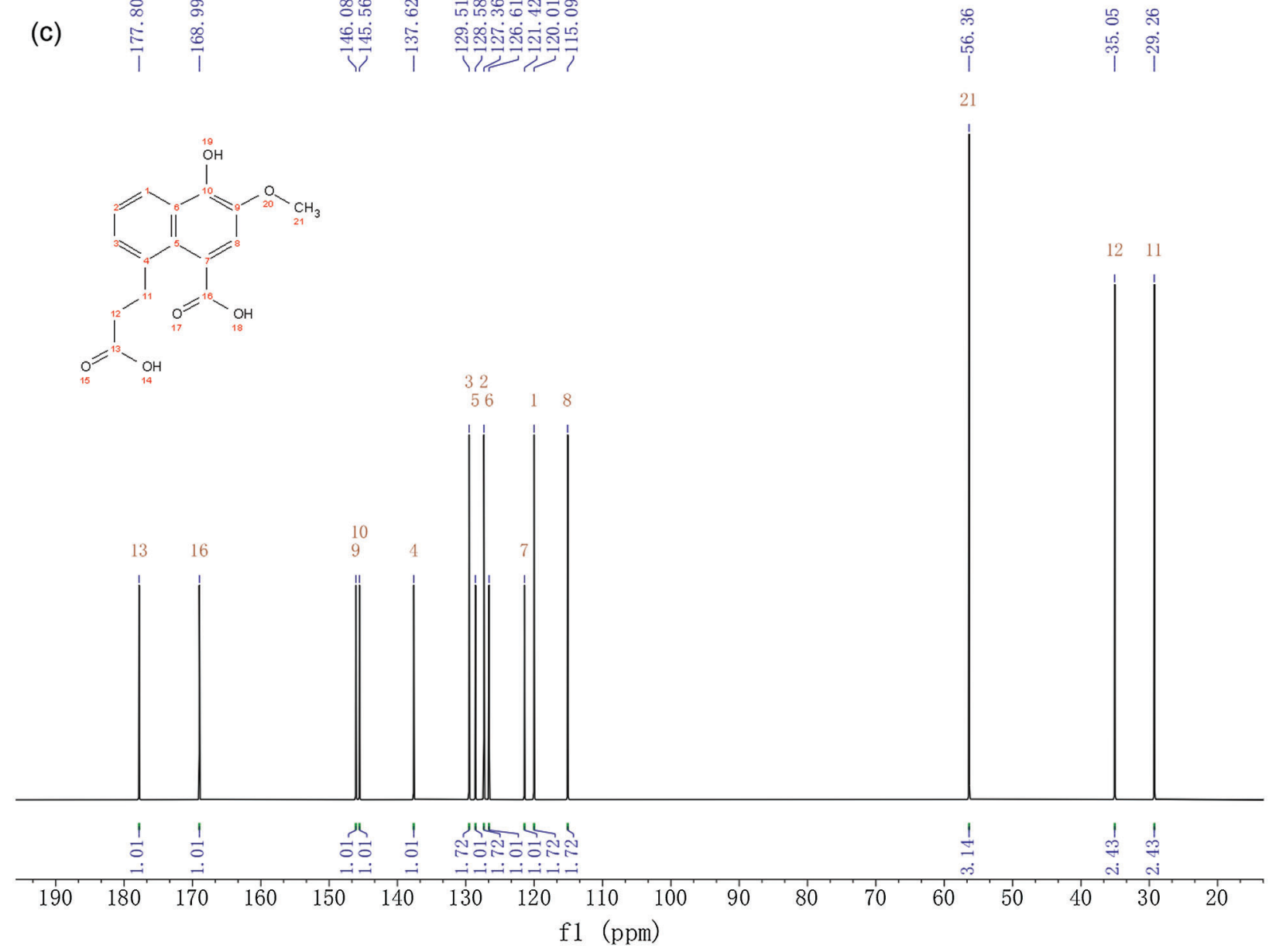

Figure 3: FTIR (a), ${ }^{1} \mathrm{H}$ NMR (b) and ${ }^{13} \mathrm{C}$ NMR (c) spectra of $\beta$-HA
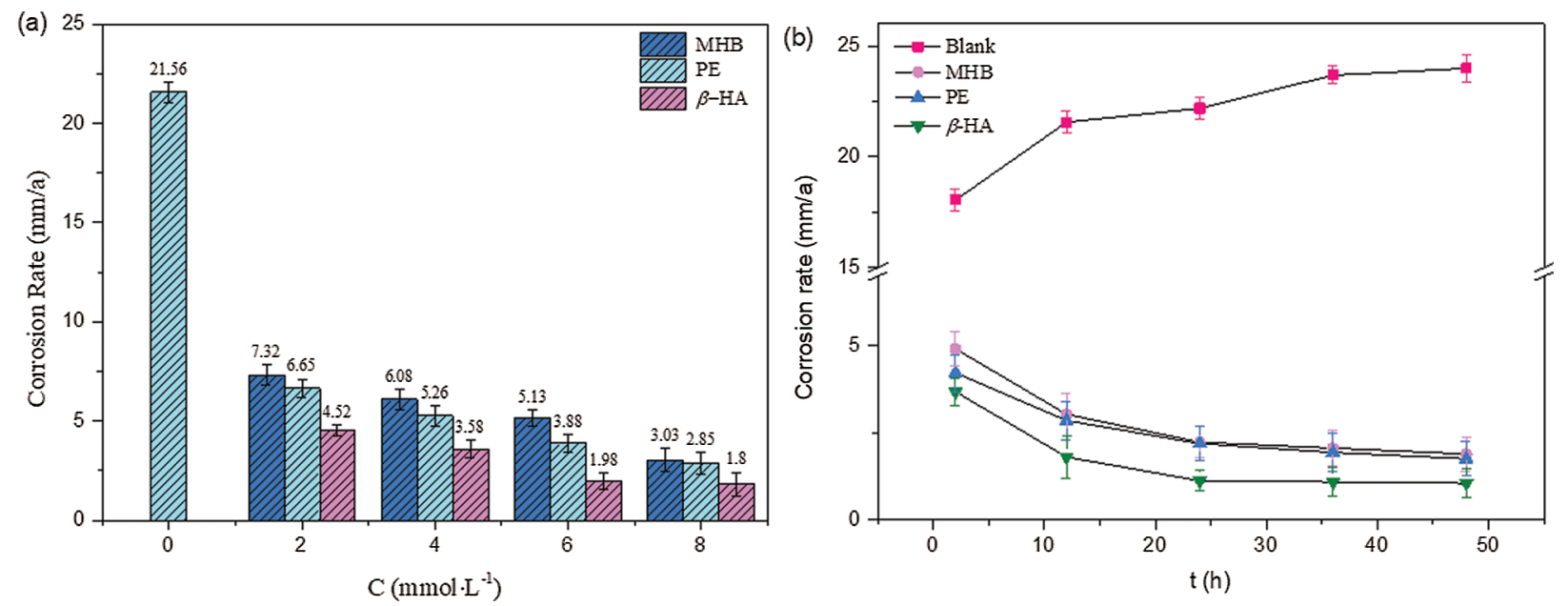

Figure 4: Corrosion rate for $\mathrm{N} 80$ steel in $15 \% \mathrm{HCl}$ solution from weight loss measurements at $303 \mathrm{~K}$ (a) $\beta$-HA concentration (b) immersion time 
Table 2: Corrosion rate and inhibition efficiency for $\mathrm{N} 80$ steel in $15 \% \mathrm{HCl}$ solution from weight loss measurements

\begin{tabular}{|c|c|c|c|c|c|c|}
\hline \multirow[t]{2}{*}{$C_{\mathrm{inh}} / \mathrm{mmol} \cdot \mathrm{L}^{-1}$} & \multicolumn{2}{|c|}{ MHB } & \multicolumn{2}{|c|}{$\mathrm{PE}$} & \multicolumn{2}{|c|}{ HA } \\
\hline & $v_{\text {corr }}(\mathrm{mm} / \mathrm{a})$ & $\eta(\%)$ & $v_{\text {corr }}(\mathrm{mm} / \mathrm{a})$ & $\eta(\%)$ & $v_{\text {corr }}(\mathrm{mm} / \mathrm{a})$ & $\eta(\%)$ \\
\hline 0 & 21.56 & - & 21.56 & - & 21.56 & - \\
\hline 2 & 7.32 & 66.05 & 6.65 & 69.16 & 4.52 & 79.04 \\
\hline 4 & 6.08 & 71.80 & 5.26 & 75.60 & 3.58 & 83.40 \\
\hline 6 & 5.13 & 76.21 & 3.88 & 82.00 & 1.98 & 90.82 \\
\hline 8 & 3.03 & 85.95 & 2.85 & 86.78 & 1.8 & 91.65 \\
\hline
\end{tabular}
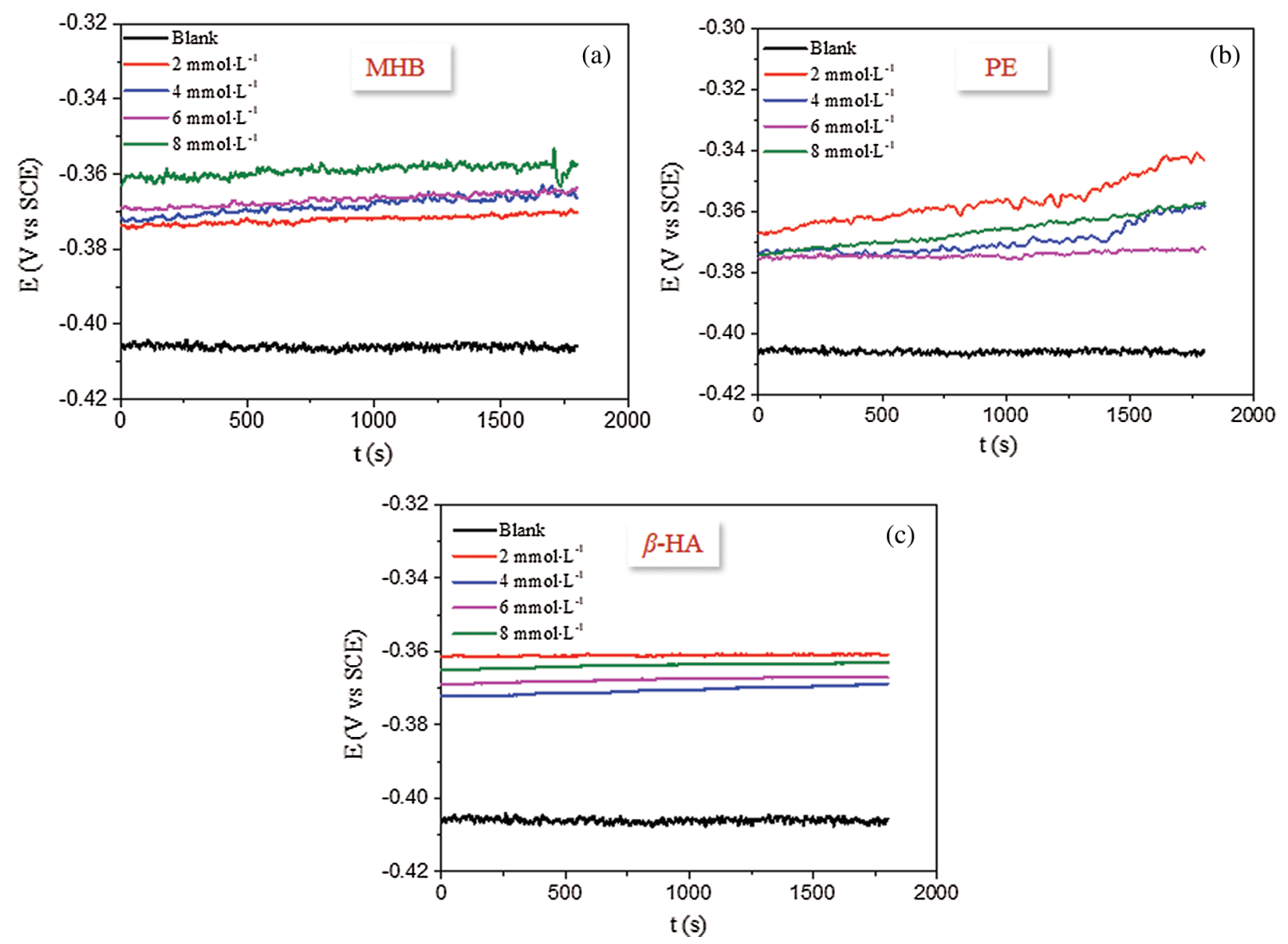

Figure 5: OCP curves of N80 steel in various test solutions: (a) MHB; (b) PE; (c) $\beta$-HA

\subsection{Potentiodynamic Polarization Measurements}

Potentiodynamic polarization curves for MHB, PE and $\beta$-HA are showed in Fig. 6 . The electrochemical parameters were attained by using Extrapolation method from the polarization curves containing corrosion 
current density $\left(i_{\text {corr }}\right)$, corrosion potential $\left(E_{\text {corr }}\right)$, anodic and cathodic Tafel slopes $\left(\beta_{\mathrm{a}}, \beta_{\mathrm{c}}\right)$, and inhibition efficiency $(\eta)$. Moreover, the value of inhibition efficiency $(\eta)$ were estimated by Eq. (10):

$\eta=\frac{i_{c o r r}^{0}-i_{\text {corr }}}{i_{\text {corr }}^{0}} \times 100$

where $i_{\text {corr }}^{0}$ and $i_{\text {corr }}$ represent the corrosion current density in the absence and presence of inhibitors, respectively.
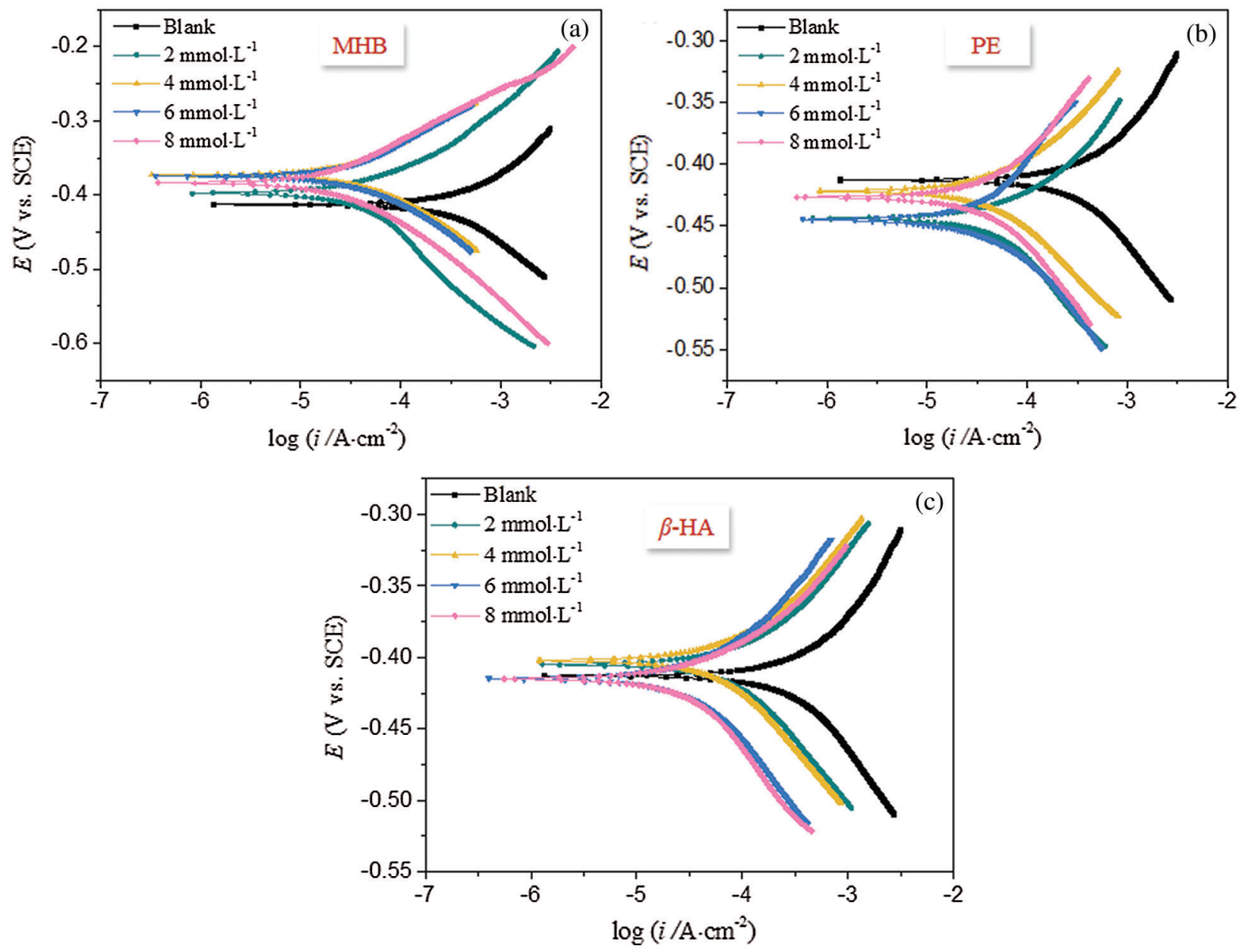

Figure 6: Polarization curves of N80 steel in various test solutions: (a) MHB; (b) PE; (c) $\beta$-HA

As indicated in Figs. $6 \mathrm{a}-6 \mathrm{c}$, the values of $E_{\mathrm{corr}}$ in the presence of the inhibitor MHB, PE and $\beta$-HA shift less than $85 \mathrm{mV}$, respectively, the three inhibitors belong to mixed-type inhibitor [36]. The polarization curve after the addition of the corrosion inhibitor moves to the left, which indicates that the corrosion current density has decreased with the inhibitor. The reduced slope of the anode and cathode displays that the inhibitors have the ability to block the anodic and cathodic reactions simultaneously. Tab. 3 shows that the values of $\beta_{\mathrm{a}}$ and $\beta_{\mathrm{c}}$ for the $15 \% \mathrm{HCl}$ solution are extremely larger than other groups, which indicates that adsorption films were formed on the N80 surface after inhibitors were added. 
The values $\beta_{\mathrm{a}}$ and $\beta_{\mathrm{c}}$ did not change much with different types of inhibitors, which means that the anodic and cathodic reactions were similar among all. The corrosion current density on the N80 electrode in 15\% $\mathrm{HCl}$ solution in the absence of inhibitor was $6.42 \times 10^{-4} \mathrm{~A} \cdot \mathrm{cm}^{-2}$. As the inhibitor concentration increases, corrosion current density $i_{\text {corr }}$ decreases and inhibition efficiency increases, which is related to the adsorption of inhibitors molecules on the N80 steel surface. The interaction between corrosion inhibitors and $\mathrm{Fe}$ atoms on the surface of carbon steel, and the rate of corrosion on the surface of N80 decreased. By comparing the corrosion current density of $\mathrm{MHB}, \mathrm{PE}$ and $\beta$-HA, the lowest value was observed at $0.38 \times 10^{-4} \mathrm{~A} \cdot \mathrm{cm}^{-2}$ for $8 \mathrm{mmol} \cdot \mathrm{L}^{-1} \beta$-HA. This indicates that the corrosion inhibition of $\beta$-HA is better than individual inhibitor.

\subsection{Electrochemical Impedance Spectroscopy Measurements}

Fig. 7 shows Nyquist and Bode plots of the $\mathrm{N} 80$ steel in $15 \% \mathrm{HCl}$ solution in the absence and presence of various concentrations of inhibitors. It can be seen from Fig. 7, the Nyquist curves of the three inhibitors show only one time constant corresponding to one capacitor circuit, indicating that the dynamics of the electrochemical system is controlled by the charge transfer process. In addition, the shape of Nyquist plots is almost similar in absence and presence of inhibitor, which suggests that the addition of corrosion inhibitor has no effect on the corrosion mechanism of N80 steel. The radius of capacitive reactance arc in Nyquist plots is below the real axis due to the roughness and uniformity of N80 steel electrode surface [37]. The radius of capacitive arc increases with the increase of inhibitor concentration in the $15 \% \mathrm{HCl}$ solution, suggesting that inhibition efficiency increases with inhibitor concentration. Compared with capacitive arcs when MHB or PE is added alone (Fig. 7d, Fig. 7g), the radius of capacitive arc of the $\beta$-HA inhibitor is obviously larger than that of MHB or PE alone. This indicates that the corrosion inhibition performance of $\beta$-HA is better than that of MHB and PE alone [38-40].
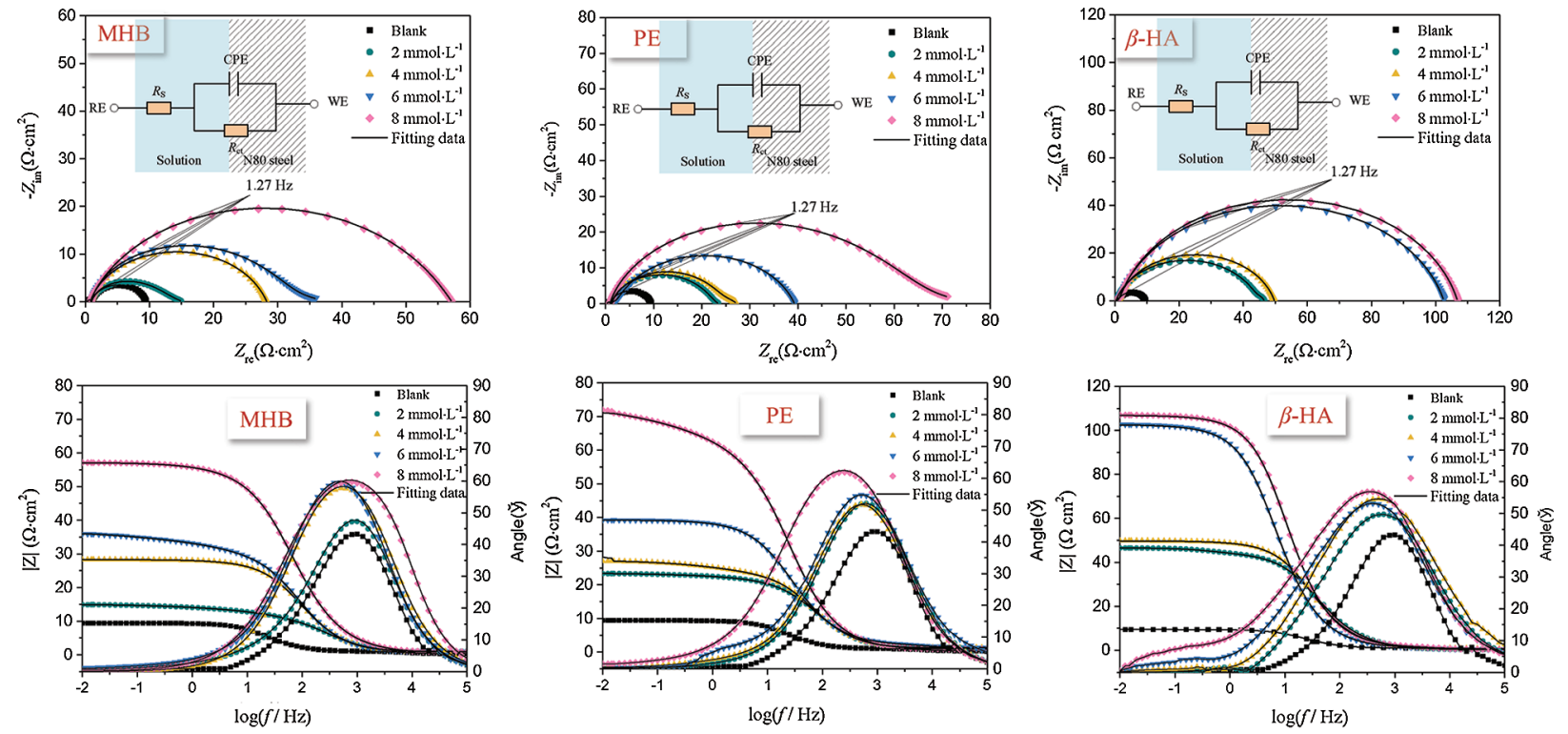

Figure 7: EIS images of N80 steel in various test solutions

Based on Bode plots, the $|Z|_{0.01 \mathrm{~Hz}}$ can be used to evaluate the corrosion rate of electrode [41]. It can be seen from Fig. $7 \mathrm{~b}$ that the $|Z|_{0.01 \mathrm{~Hz}}$ is about $10 \Omega \cdot \mathrm{cm}^{-2}$ when $\mathrm{N} 80$ steel was immersed into $15 \% \mathrm{HCl}$ solution for $12 \mathrm{~h}$. As the concentration of inhibitor MHB, PE and $\beta$-HA increased respectively, the $|Z|_{0.01 \mathrm{~Hz}}$ presented an increase trend. When the concentration of $\beta$-HA is $8 \mathrm{mmol} \cdot \mathrm{L}^{-1}$, the $|Z|_{0.01 \mathrm{~Hz}}$ increase to $104 \Omega \cdot \mathrm{cm}^{-2}$. 
Table 3: The corrosion kinetic parameters of $\mathrm{N} 80$ steel after soaking in $15 \% \mathrm{HCl}$ solution with different concentration of corrosion inhibitor at $303 \mathrm{~K}$

\begin{tabular}{lllllll}
\hline Corrosion inhibitor & $\begin{array}{l}C \\
\left(\mathrm{mmol} \cdot \mathrm{L}^{-1}\right)\end{array}$ & $\begin{array}{l}b_{\mathrm{a}} / \\
\left(\mathrm{mV} \cdot \mathrm{dec}^{-1}\right)\end{array}$ & $b_{\mathrm{c}} /\left(\mathrm{mV} \cdot \mathrm{dec}^{-1}\right)$ & $\begin{array}{l}E_{\mathrm{corr}} / \\
(\mathrm{V} v s . \mathrm{SCE})\end{array}$ & $\begin{array}{l}i_{\text {corr }} \times 10^{4} / \\
\left(\mathrm{A} \cdot \mathrm{cm}^{-2}\right)\end{array}$ & $\eta / \%$ \\
\hline Blank & 0 & 152.3 & -180.4 & -0.42 & 6.42 & - \\
MHB & 2 & 89.1 & -141.2 & -0.44 & 1.63 & 74.61 \\
& 4 & 123.3 & -135.0 & -0.41 & 1.34 & 79.13 \\
& 6 & 168.9 & -109.6 & -0.45 & 1.19 & 81.46 \\
& 8 & 83.2 & -97.0 & -0.43 & 1.10 & 82.87 \\
PE & 2 & 91.3 & -123.1 & -0.40 & 2.06 & 67.91 \\
& 4 & 84.1 & -114.3 & -0.41 & 1.69 & 73.67 \\
& 6 & 84.5 & -125.3 & -0.42 & 1.01 & 84.27 \\
& 6 -HA & 67.2 & -126.9 & -0.42 & 0.87 & 86.45 \\
& 2 & 94.2 & -173.8 & -0.41 & 1.42 & 77.88 \\
& 4 & 95.6 & -88.1 & -0.38 & 0.98 & 84.74 \\
& 6 & 95.3 & -91.1 & -0.39 & 0.60 & 90.65 \\
& 8 & 78.9 & -90.3 & -0.38 & 0.38 & 94.08 \\
\hline
\end{tabular}

In order to better understand the corrosion reaction process, the equivalent circuit model (Fig. 7) are used to fit EIS data, the fitting parameter was presented in Tab. 4. Among them, $R_{\mathrm{s}}$ is the solution resistance, $R_{\mathrm{ct}}$ is the charge transfer resistance, $\mathrm{CPE}$ was the constant phase angle element, representing the capacitive property of electric double layer. The value of $C_{\mathrm{dl}}$ can be calculated by Eq. (11) [42], the corrosion inhibition efficiency $(\eta)$ and surface coverage $(\theta)$ could be obtained according to the Eqs. (12) and (13), respectively [43]:

$C_{\mathrm{dl}}=\left(Y_{0} R_{\mathrm{ct}}^{1-n}\right)^{\frac{1}{n}}$

$\eta=\frac{R_{\mathrm{ct}}-R_{\mathrm{ct}}^{0}}{R_{\mathrm{ct}}} \times 100$

$\theta=\frac{R_{\mathrm{ct}}-R_{\mathrm{ct}}^{0}}{R_{\mathrm{ct}}}$

where, the $R_{c t}^{0}$ is the charge transfer resistance without inhibitor, $R_{c t}$ is the charge transfer resistance with inhibitor obtained by electrochemical impedance spectroscopy data.

As shown in Tab. 4, the change of $R_{\mathrm{S}}$ is not obvious in different corrosion environments. However, the film resistance $R_{\mathrm{f}}$ and $R_{\mathrm{ct}}$ values show an increasing trend after adding inhibitors MHB, PE, or $\beta$-HA, indicating the inhibitor molecules are adsorbed on the sample surface to form a protective film. By comparison, arc radius of capacitive resistance and film coverage are the largest when the concentration of $\beta$-HA is $8 \mathrm{mmol} \cdot \mathrm{L}^{-1}$, respectively (Tab. 4). These results also illustrate that $\beta$-HA is better than individual inhibitor for corrosion prediction. Moreover, the change trend of $C_{\mathrm{dl}}$ was opposite to that of $R_{\mathrm{ct}}$ value with the increase of inhibitor concentration. 
Table 4: Electrochemical parameters of $\mathrm{N} 80$ steel in the absence and presence of inhibitor in $15 \% \mathrm{HCl}$ solution at $303 \mathrm{~K}$ obtained from equivalent electrical circuit

\begin{tabular}{|c|c|c|c|c|c|c|c|c|c|}
\hline $\begin{array}{l}\text { Corrosion } \\
\text { inhibitor }\end{array}$ & $\begin{array}{l}C \\
/ \mathrm{mmol} \cdot \mathrm{L}^{-1}\end{array}$ & $\begin{array}{l}R_{\mathrm{s}} \\
/ \Omega \cdot \mathrm{cm}^{2}\end{array}$ & $\begin{array}{l}Y_{0} \\
/ \mathrm{F} \cdot \mathrm{cm}^{-2}\end{array}$ & $\begin{array}{l}R_{\mathrm{ct}} \\
/ \Omega \cdot \mathrm{cm}^{2}\end{array}$ & $n$ & $\begin{array}{l}C_{d l} \\
/ \mu \mathrm{F} \cdot \mathrm{cm}^{-2}\end{array}$ & $\theta$ & $\begin{array}{l}\eta \\
1 \%\end{array}$ & $\begin{array}{l}\text { Chi- } \\
\text { square }\end{array}$ \\
\hline Blank & 0 & 0.342 & 0.00190 & 9.1 & 0.76 & 535.34 & - & - & $1.1 \times 10^{-4}$ \\
\hline \multirow[t]{4}{*}{ MHB } & 2 & 1.01 & 0.00034 & 14.12 & 0.89 & 184.52 & 0.3557 & 35.57 & $1.3 \times 10^{-4}$ \\
\hline & 4 & 0.85 & 0.00020 & 27.45 & 0.91 & 121.11 & 0.6685 & 66.85 & $1.1 \times 10^{-4}$ \\
\hline & 6 & 0.94 & 0.00016 & 36.58 & 0.89 & 86.75 & 0.7512 & 75.12 & $3.0 \times 10^{-4}$ \\
\hline & 8 & 0.90 & 0.00011 & 56.25 & 0.86 & 49.88 & 0.8382 & 83.82 & $2.2 \times 10^{-4}$ \\
\hline \multirow[t]{4}{*}{ PE } & 2 & 1.19 & 0.00035 & 22.38 & 0.90 & 212.58 & 0.5934 & 59.34 & $1.5 \times 10^{-4}$ \\
\hline & 4 & 1.30 & 0.00046 & 26.03 & 0.78 & 134.98 & 0.6504 & 65.04 & $2.1 \times 10^{-4}$ \\
\hline & 6 & 1.31 & 0.00039 & 37.94 & 0.69 & 61.70 & 0.7601 & 76.01 & $1.8 \times 10^{-4}$ \\
\hline & 8 & 0.90 & 0.00030 & 75.10 & 0.68 & 54.37 & 0.8788 & 87.88 & $1.3 \times 10^{-4}$ \\
\hline \multirow[t]{4}{*}{$\beta$-HA } & 2 & 0.32 & 0.00021 & 46.25 & 0.83 & 83.56 & 0.8032 & 80.32 & $1.2 \times 10^{-4}$ \\
\hline & 4 & 0.72 & 0.00018 & 49.04 & 0.81 & 62.53 & 0.8144 & 81.44 & $2.0 \times 10^{-4}$ \\
\hline & 6 & 0.42 & 0.00013 & 102.18 & 0.81 & 49.35 & 0.9109 & 91.09 & $1.8 \times 10^{-4}$ \\
\hline & 8 & 0.44 & 0.00009 & 106.53 & 0.78 & 24.75 & 0.9146 & 91.46 & $2.3 \times 10^{-4}$ \\
\hline
\end{tabular}

The corrosion inhibition efficiency $(\eta)$ can be obtained by Eq. (14). In the case of MHB and PE added alone, the $\eta$ value can be up to $83.82 \%$ and $87.88 \%$ when the adding concentration is $8 \mathrm{mmol} \cdot \mathrm{L}^{-1}$. However, the $\eta$ values in the case of $\beta$-HA were greatly enhanced as the injection concentration increased. When the concentration of $\beta$-HA is $8 \mathrm{mmol} \cdot \mathrm{L}^{-1}$, the $\eta$ values reached up to $91.46 \%$. Therefore, the corrosion inhibition effect of $\beta$-HA was stronger than that of MHB and PE added alone, suggesting that the corrosion rate of $\mathrm{N} 80$ steel in $15 \% \mathrm{HCl}$ solution is further reduced and the corrosion inhibition performance is increased. Also, it is important to note that the weight loss results are in conformity with the weight loss results.

\subsection{Adsorption Isotherm}

The experimental data were fitted with adsorption models, and the Langmuir model Eq. (14) has the best fit. The Gibbs free energy $\left(\Delta G_{\text {ads }}\right)$ for the adsorbent is obtained from electrochemical impedance spectroscopy results, as shown in Eq. (15) [44].

$\frac{c_{\mathrm{inh}}}{\theta}=c_{\mathrm{inh}}+\frac{1}{K_{\mathrm{ads}}}$

$\Delta G_{\mathrm{ads}}=-R T \ln \left(55.5 K_{a d s}\right)$

where the $K_{\text {ads }}$ is the equilibrium constant of the adsorption, $C_{\text {inh }}$ is the inhibitor concentration and $\theta$ is the film coverage. $R$ is the mole constant of gas with unit of $\mathrm{J} \cdot \mathrm{mol}^{-1} \cdot \mathrm{K}^{-1}$ and $T$ is the temperature with unit of Kelvin, and the concentration of water molecules is $55.5 \mathrm{~mol} \cdot \mathrm{L}^{-1}$.

The linear $C / \theta \sim C$ curves fitting by the Langmuir model are shown in Fig. 8, and the value of $K_{\text {ads }}$ are 208.3, 555.5 and 1666.7 with a high correlation coefficient of $0.9927,0.9865$ and 0.9932 , respectively. Therefore, the adsorption behavior of MHB, PE and $\beta$-HA obey the Langmuir adsorption isotherm. The $\Delta G_{\text {ads }}$ can reflect the adsorption type of corrosion inhibitor on N80 steel surface, and it is related to $K_{\text {ads }}$. $\Delta G_{\text {ads }}$ is $-20 \mathrm{~kJ} \cdot \mathrm{mol}^{-1}$ or more negative, the corrosion inhibitor molecules are adsorbed on the metal surface by electrostatic interaction, which is physical adsorption; while $\Delta G_{\text {ads }}$ is $-40 \mathrm{~kJ} \cdot \mathrm{mol}^{-1}$ or more 
negative, it belongs to chemical adsorption because of the covalent bond formed by electron transfer or sharing. Using Eq. (16), the $\Delta G_{\text {ads }}$ were $-23.57,-26.04$ and $-28.81 \mathrm{~kJ} \cdot \mathrm{mol}^{-1}$ under the standard atmospheric pressure, respectively, which indicates that the adsorption of MHB, PE and $\beta$-HA on N80 steel surface could be the mixed adsorption process that involves both physical and chemical adsorption. By comparing outcomes of experiments, the adsorption force of $\beta$-HA is stronger than MHB and PE, and adsorption cannot be easily reversed. The adsorption is exothermic because $\Delta G_{\text {ads }}$ is smaller than zero [45].

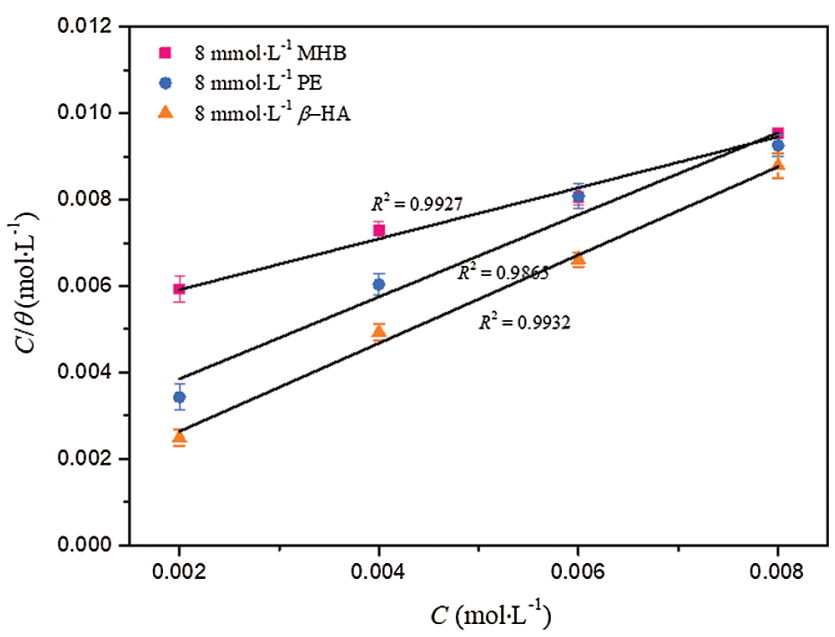

Figure 8: $\mathrm{C} / \theta \sim \mathrm{C}$ plots of $\mathrm{N} 80$ steel after $12 \mathrm{~h}$ immersion in corrosion solution with different inhibitors at $303 \mathrm{~K}$

In order to further understand the adsorption of corrosion inhibitor at the metal/solution interface, the adsorption entropy of activation $\left(\Delta S^{*}\right)$ and enthalpy of activation $\left(\Delta H^{*}\right)$ were introduced. Based on the transition state equation, $\Delta S^{*}$ and $\Delta H^{*}$ are be obtained [15].

$v_{\text {corr }}=\frac{R T}{N H} \exp \left(\frac{\Delta S^{*}}{R}\right) \exp \left(\frac{-\Delta H^{*}}{R T}\right)$

The plots of $\log \left(v_{\text {corr }} / T\right) v s .1 / T$ yield a straight line, as shown in Fig. 9, the values of $\Delta H^{*}$ and $\Delta S^{*}$ can be determined according to the slope and intercept of linear fitting. The obtained values are listed in Tab. 5. It can be clearly seen from Tab. 5 that the value of $\Delta H^{*}$ is positive, which indicates that the adsorption of corrosion inhibitor on the metal interface is endothermic process, that is to say, the interaction between inhibitor molecules and $\mathrm{Fe}$ atoms on metal surface is more favorable at high temperature, and the adsorption of corrosion inhibitor molecules on the surface of N80 steel is easier. Due to the negative $\Delta S^{*}$ value, the adsorption process is entropy increasing. The results suggest that the adsorption of corrosion inhibitor molecules is greater than desorption in the rate determining step. The increase of adsorption substances leads to the increase of solution confusion, and the active substances adsorb on the surface of N80 steel instead of water molecules to form an adsorption layer to inhibit the corrosion of N80 steel.

\subsection{Adsorption Rate}

The surface coverage of $\mathrm{N} 80$ steel sample in $\mathrm{HCl}$ solution with $8 \mathrm{mmol} \cdot \mathrm{L}^{-1}$ concentration of $\mathrm{MHB}, \mathrm{PE}$ and $\beta$-HA at $303 \mathrm{~K}$ is given in Fig. 10. 
JRM, 2022, vol.10, no.2

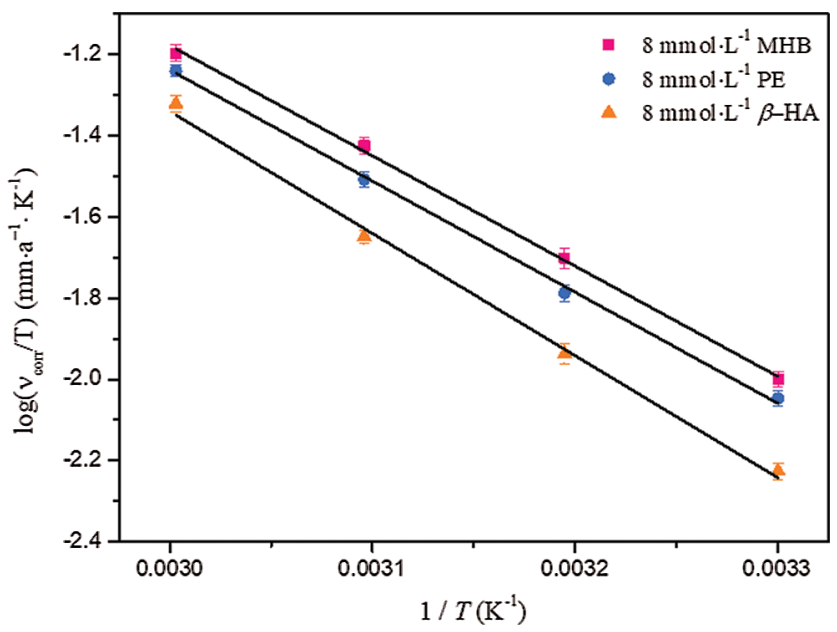

Figure 9: The relationship between $\log \left(v_{\text {corr }} / T\right)$ and $(1 / \mathrm{T})$ for $\mathrm{N} 80$ steel in $15 \% \mathrm{HCl}$ solution with MHB PE and $\beta$-HA

Table 5: Thermodynamic parameters of adsorption for $\mathrm{N} 80$ steel in $15 \% \mathrm{HCl}$ solution adding corrosion inhibitor MHB, PE and $\beta$-HA, respectively

\begin{tabular}{lllllll}
\hline Inhibitor & \multicolumn{4}{c}{ Corrosion rate $\left(\mathrm{mm} \cdot \mathrm{a}^{-1}\right)$} & $\Delta H\left(\mathrm{~kJ} \cdot \mathrm{mol}^{-1}\right)$ & $\Delta S\left(\mathrm{~J} \cdot \mathrm{mol}^{-1} \cdot \mathrm{K}^{-1}\right)$ \\
\cline { 2 - 5 } & $303 \mathrm{~K}$ & $313 \mathrm{~K}$ & $323 \mathrm{~K}$ & $333 \mathrm{~K}$ & & \\
\hline MHB & 3.03 & 6.23 & 12.15 & 21.18 & 52.00161 & -115.456 \\
PE & 2.85 & 5.11 & 10.03 & 19.16 & 52.36791 & -115.512 \\
$\beta$-HA & 1.8 & 3.61 & 7.25 & 15.89 & 57.54238 & -101.943 \\
\hline
\end{tabular}

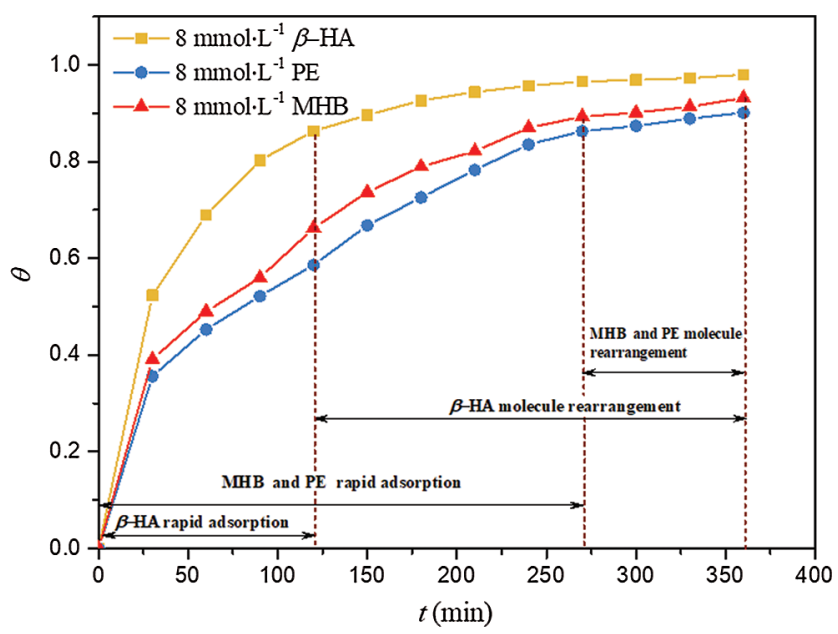

Figure 10: The relationship between $\theta$ and time on the N80 steel surface after $6 \mathrm{~h}$ immersion in $15 \% \mathrm{HCl}$ solution with $8 \mathrm{mmol} \cdot \mathrm{L}^{-1} \mathrm{MHB}, \mathrm{PE}$ and $\beta$-HA, respectively 
The whole adsorption process is divided into two stages: the first stage is a rapid adsorption process from $0 \sim 120 \mathrm{~min}$ for $\beta$-HA, and the second stage is a rearrangement stage from 120-360 min, while the rapid adsorption process of MHB and PE is from 0 to $270 \mathrm{~min}$, suggesting that that the film forming speed of inhibitor molecule $\beta$-HA is relatively fast. The behavior is similar with other self-assembled films [46,47]. In the first stage, the benzene ring and the organic functional aldehyde groups in the inhibitor molecule play a major role. Among them, the aldehyde group rapidly attach to metal surface via chemical bonding. In the meantime, the benzene ring has a hydrophobic structure and generates a hydrophobic film. In the second stage, large $\pi$ bond of benzene ring is combined with the empty orbital of Fe atoms to form more stable bonds, resulting in a stronger adsorption and denser film [48].

\subsection{Scanning Electron Microscopy}

SEM analysis was performed to obtain deeper understanding about the corrosion morphology of $\mathrm{N} 80$ steel immersed in $15 \% \mathrm{HCl}$ solution in absence and presence of inhibitor at $303 \mathrm{~K}$. Fig. 11a shows the morphology of $\mathrm{N} 80$ steel after immersing in $15 \% \mathrm{HCl}$ solution without inhibitor for $12 \mathrm{~h}, \mathrm{~N} 80$ steel surface was severely damaged with cracks and pits. Figs. $11 \mathrm{~b}$ and $11 \mathrm{c}$ show the morphology after being soaked into the solution with $8 \mathrm{mmol} \cdot \mathrm{L}^{-1} \beta$-HA corrosion inhibitors. Compared with Fig. 11a, there were no obvious corrosion marks and serious corrosion pits on the metal surface. However, some small cracks still existed under a high magnification, indicating that the area covered by the corrosion inhibitor was incomplete. After adding inhibitor $\beta$-HA into the $15 \%$ hydrochloric acid solution, a layer of white coating appears on the surface of the sample, as shown in Fig. 11d. It was noted that the white coatings are unevenly distributed in an overlapping pattern and cracks are greatly decreased in this case. This indicates that the $\beta$-HA corrosion inhibitor can effectively slow down the corrosion by increasing the surface film coverage.
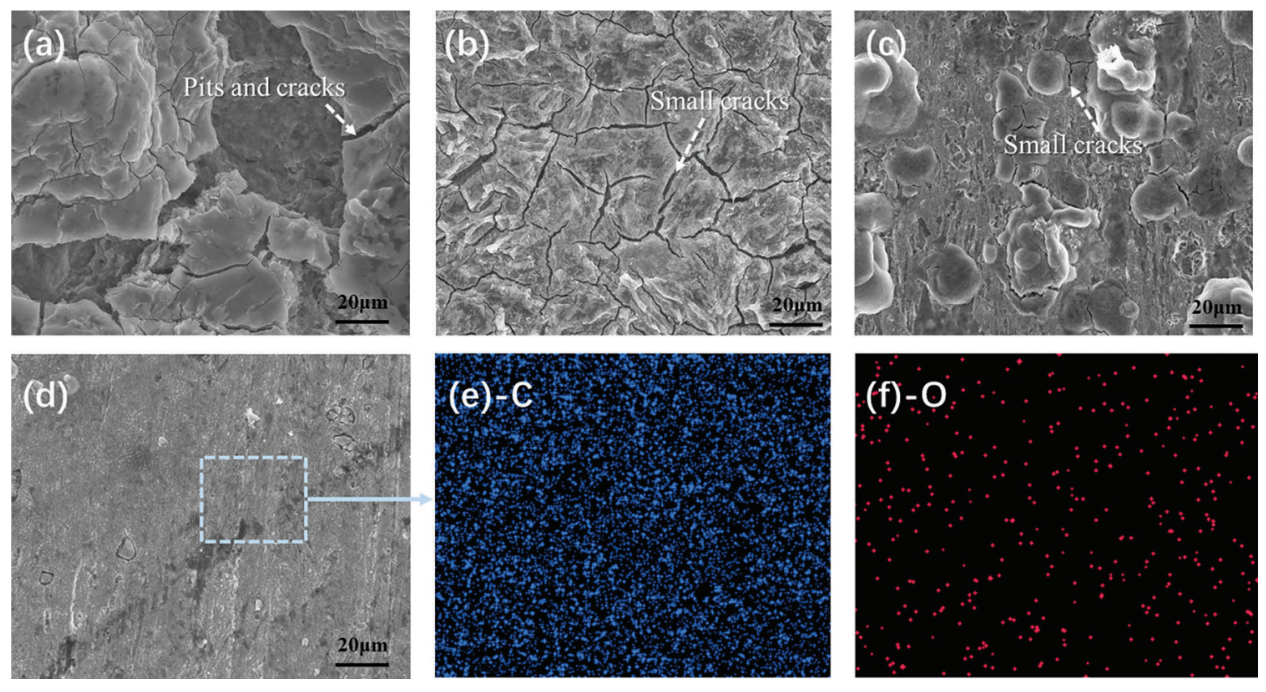

Figure 11: Corrosion morphology and element distribution of $\mathrm{N} 80$ steel in various solutions (a) $15 \% \mathrm{HCl}$ solution-12 h (b) $15 \% \mathrm{HCl}+8 \mathrm{mmol} \cdot \mathrm{L}^{-1} \mathrm{MHB}$ solution- $12 \mathrm{~h}$ (c) $15 \% \mathrm{HCl}+8 \mathrm{mmol} \cdot \mathrm{L}^{-1} \mathrm{PE}$ solution- $12 \mathrm{~h}$ (d) $15 \% \mathrm{HCl}+8 \mathrm{mmol} \cdot \mathrm{L}^{-1} \beta$-HA-12 $\mathrm{h}$; and (e) $\mathrm{C}$; (f) $\mathrm{O}$ distribution

\subsection{D Morphology}

The 3D morphology of $\mathrm{N} 80$ samples were immersed in $15 \% \mathrm{HCl}$ for $12 \mathrm{~h}$ at $303 \mathrm{~K}$ without and with inhibitors are shown in Fig. 12. 

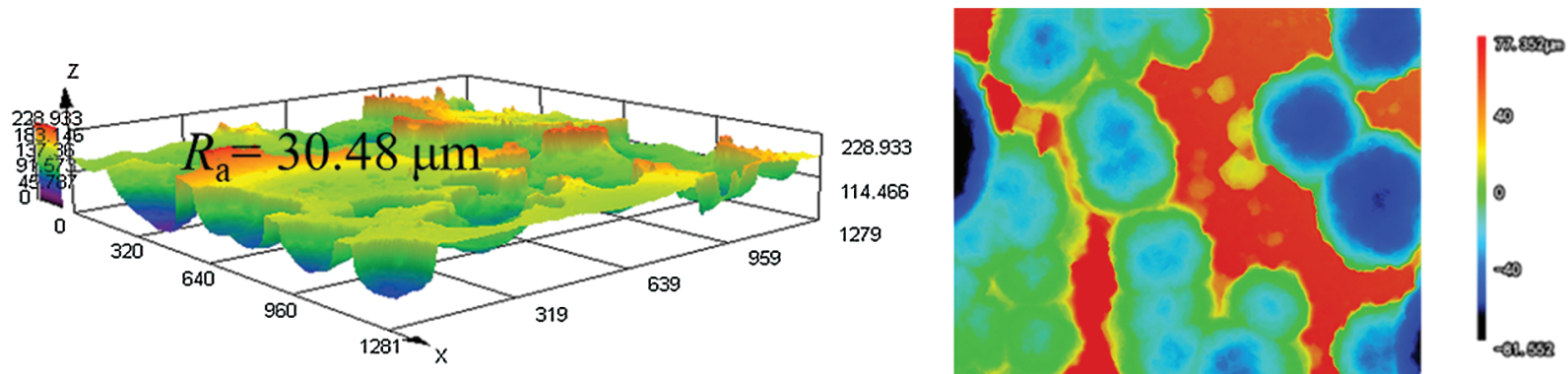

(a)
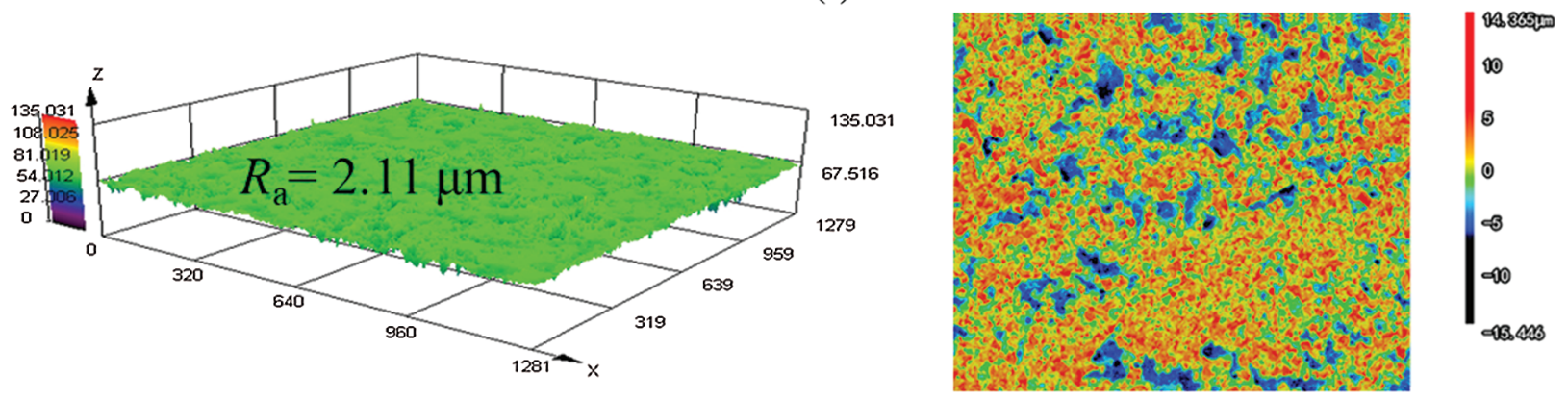

(b)
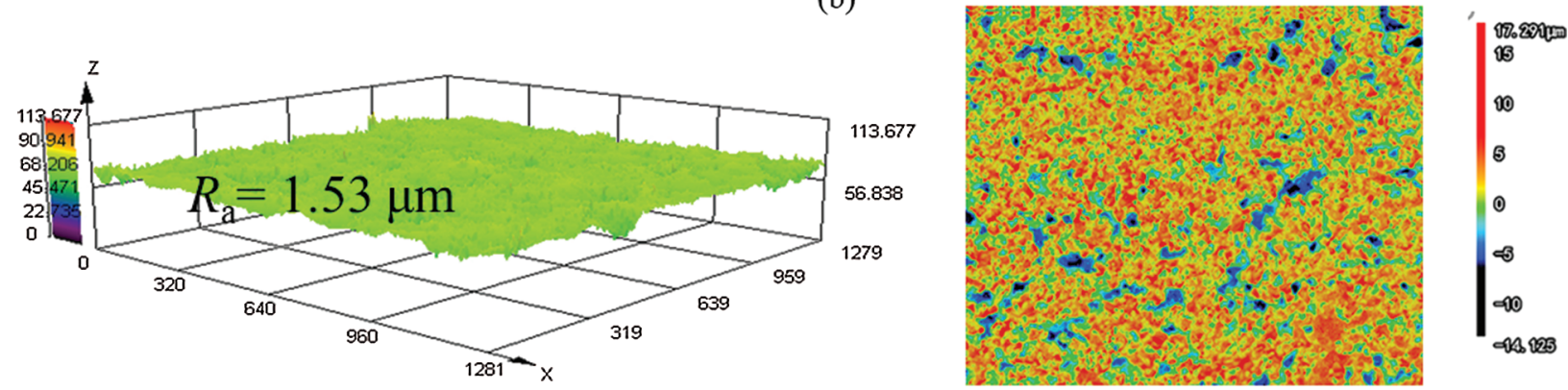

(c)
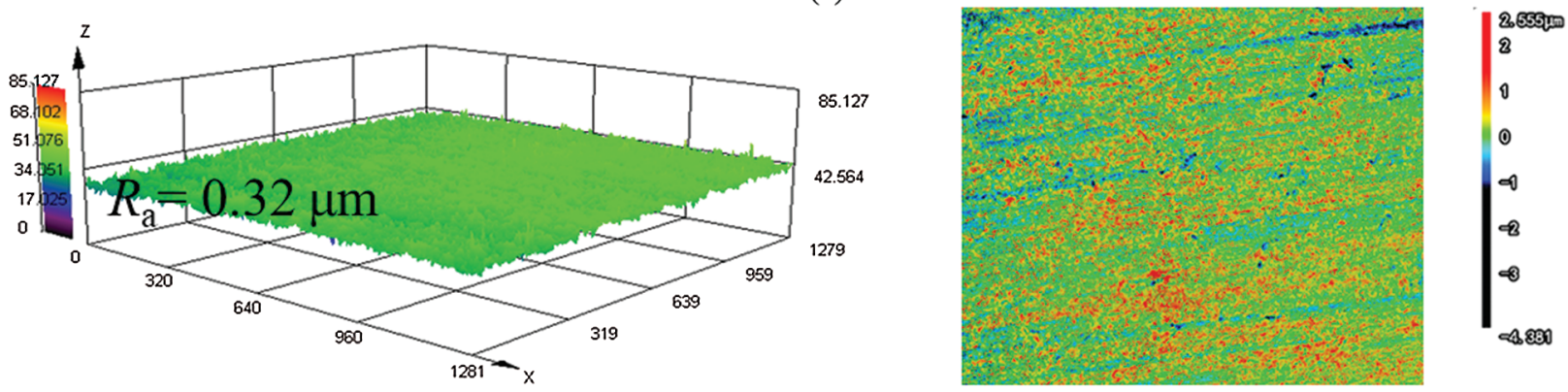

(d)

Figure 12: 3D corrosion morphology and roughness of $\mathrm{N} 80$ steel in various solutions (a) $15 \% \mathrm{HCl}$ solution-12 h (b) $15 \% \mathrm{HCl}+8 \mathrm{mmol} \cdot \mathrm{L}^{-1} \mathrm{MHB}$ solution- $12 \mathrm{~h}$ (c) $15 \% \mathrm{HCl}+8 \mathrm{mmol} \cdot \mathrm{L}^{-1} \mathrm{PE}$ solution- $12 \mathrm{~h}$ (d) $15 \% \mathrm{HCl}+8 \mathrm{mmol} \cdot \mathrm{L}^{-1} \beta$-HA-12 $\mathrm{h}$

As shown in Fig. 12a, the surface of $\mathrm{N} 80$ samples in the $15 \% \mathrm{HCl}$ solution without corrosion inhibitor was irregular and occurred severe corrosion, then the average roughness value reached up to $30.48 \mu \mathrm{m}$. After being soaked into the solution with $8 \mathrm{mmol} \cdot \mathrm{L}^{-1} \mathrm{MHB}, 8 \mathrm{mmol} \cdot \mathrm{L}^{-1} \mathrm{PE}$ and $8 \mathrm{mmol} \cdot \mathrm{L}^{-1} \beta$-HA inhibitors, the surface of the N80 samples became more and more smoother, which suggests that the corrosion process of 
N80 steel was hindered in the corrosive medium. According to Figs. 12b-12d, the average roughness values of $\mathrm{N} 80$ steel are $2.11 \mu \mathrm{m}, 1.53 \mu \mathrm{m}$ and $0.32 \mu \mathrm{m}$, respectively. The results show that the density of $\beta$-HA inhibition film is better than that of MHB and PE.

\subsection{X-Ray Photoelectron Spectroscopy}

The XPS results are shown in Fig. 13. The elements on the N80 surface are consistent with the composition given in Tab. 1.
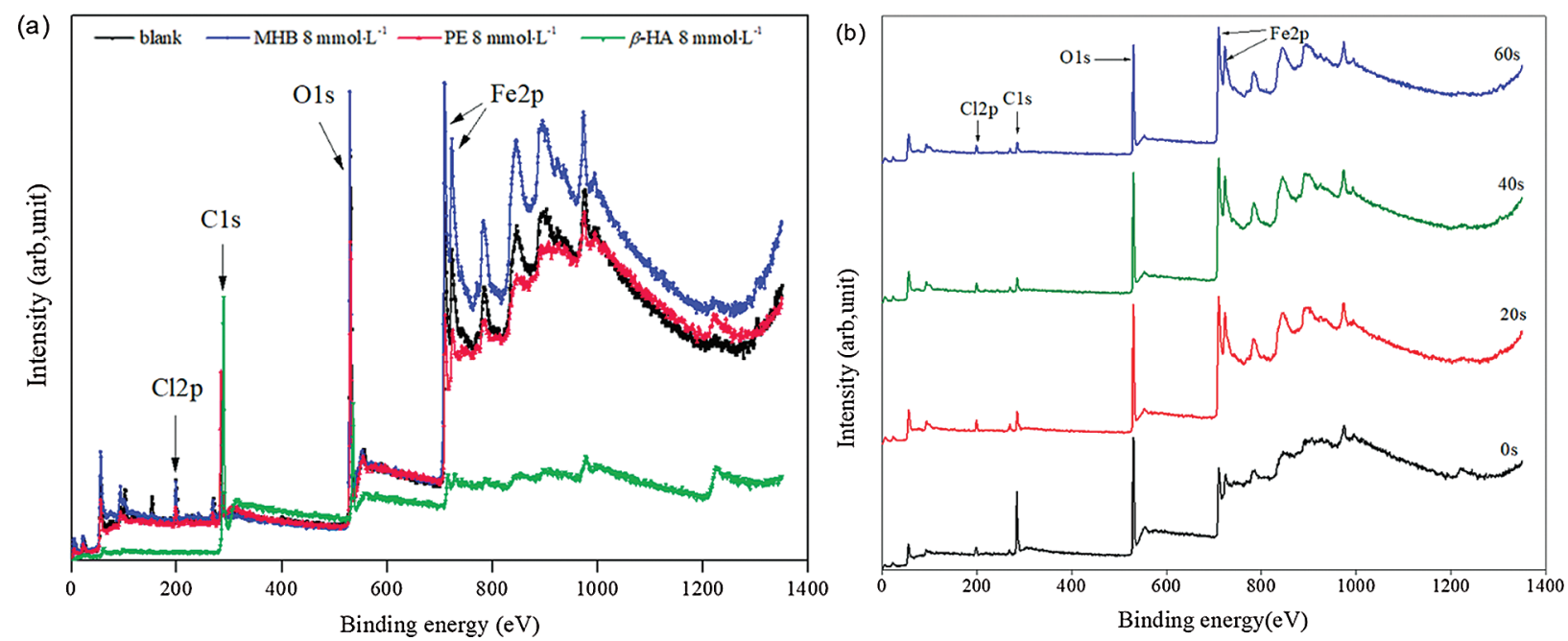

Figure 13: (a) XPS results of the N80 steel with inhibitors in $15 \% \mathrm{HCl}$ solution; (b) The binding energies along sputtering time of $\mathrm{N} 80$ steel in $15 \% \mathrm{HCl}$ with $8 \mathrm{mmol} \cdot \mathrm{L}^{-1} \beta$-HA

After the corrosion test, the content of chlorine becomes an indicator for the effect of corrosion inhibitors. As shown in Tab. 6, the content of chlorine on the metal surface was $9.51 \%$ for the blank hydrochloric acid solution without addition of any inhibitor. The content was greatly reduced when there is $\beta$-HA in the solution, and the lowest one was observed in the condition of $8 \mathrm{mmol} \cdot \mathrm{L}^{-1} \beta$-HA. This implies that $\beta$-HA inhibitor is the best among other concentrations. Fig. 13b is the binding energies along with increased sputtering time during the XPS measurements. It suggests that the coating on the surface of N80 steel has been basically stabilized.

Table 6: The content of $\mathrm{Cl} 2 \mathrm{p}$ with $\beta$-HA inhibitor in different concentrations with unit $\mathrm{mmol} \cdot \mathrm{L}^{-1}$

\begin{tabular}{lllll}
\hline Name & blank & $\begin{array}{l}\text { MHB } \\
\left(8 \mathrm{mmol} \cdot \mathrm{L}^{-1}\right)\end{array}$ & $\begin{array}{l}\mathrm{PE} \\
\left(8 \mathrm{mmol} \cdot \mathrm{L}^{-1}\right)\end{array}$ & $\begin{array}{l}\beta \text {-HA } \\
\left(8 \mathrm{mmol} \cdot \mathrm{L}^{-1}\right)\end{array}$ \\
\hline $\mathrm{Cl} 2 \mathrm{p}(\mathrm{wt} \%)$ & 9.51 & 5.95 & 3.24 & 0.09 \\
\hline
\end{tabular}

In order to account for the composition of the film more clearly, the peaks of $\mathrm{C} 1 \mathrm{~s}, \mathrm{O} 1 \mathrm{~s}, \mathrm{Fe} 2 \mathrm{p}$ and $\mathrm{Cl} 2 \mathrm{p}$ were fitted. In Fig. 14a, there are two peaks in $\mathrm{C} 1 \mathrm{~s}$, located in $284.60 \mathrm{eV}$ and $288.20 \mathrm{eV}$. The binding energy at $284.60 \mathrm{eV}$ corresponds to the $\mathrm{C}-\mathrm{H}$ bond in the $\beta$-HA inhibitor. The binding energy at $288.20 \mathrm{eV}$ is due to the $\mathrm{C}-\mathrm{O}$ bond or $-\mathrm{CHO}$ bond. The binding energy at $529.68 \mathrm{eV}$ and $531.0 \mathrm{eV}$ comes from the $\mathrm{O} 1 \mathrm{~s}$. The energy at $529.68 \mathrm{eV}$ is the dissolved oxygen, and the one at $531.0 \mathrm{eV}$ indicates the $\mathrm{C}-\mathrm{O}$ bond or $-\mathrm{CHO}$ bond. There are three principal peaks and some oscillating peaks appearing in the single peak of $\mathrm{Fe}$ 
element. Referring to XPS literature of the compounds with different valence of Fe elements [49-51], the binding energies at $710.9 \mathrm{eV}$ and $724.4 \mathrm{eV}$ in Fig. $14 \mathrm{c}$ correspond to the $\mathrm{Fe} 2 \mathrm{p} 3$ and $\mathrm{Fe} 2 \mathrm{p} 1$ in $\mathrm{Fe}_{2} \mathrm{O}_{3}$. The peak at $718.0 \mathrm{eV}$ is related to the Fe2p1 in elemental iron. In Fig. 14d, Cl2p shows two main peaks at $198.15 \mathrm{eV}$ and $199.71 \mathrm{eV}$. The peak at $198.15 \mathrm{eV}$ represents the chloride ion in the etching solution, and the binding energy at $199.71 \mathrm{eV}$ represents the $\mathrm{C}-\mathrm{Cl}$ bond. Because of the low content of chlorine, the C$\mathrm{Cl}$ peak intensity is lower. Therefore, it may be indicated that the film mainly consists of corrosion products such as iron oxide or ferrous hydroxide (film) and adsorbed organic functional groups. In the group without corrosion inhibitor, the $\mathrm{C}-\mathrm{Cl}$ peak was the strongest due to the high chlorine content, and the corrosion products were mainly iron chloride/oxide. In the groups that only single corrosion inhibitor was added, the organic functional group detected is weaker and the $\mathrm{C}-\mathrm{Cl}$ peak intensity is stronger than the $\beta$-HA inhibitor. The corrosion products were mainly iron chloride/oxide and a small amount of adsorption functional groups.
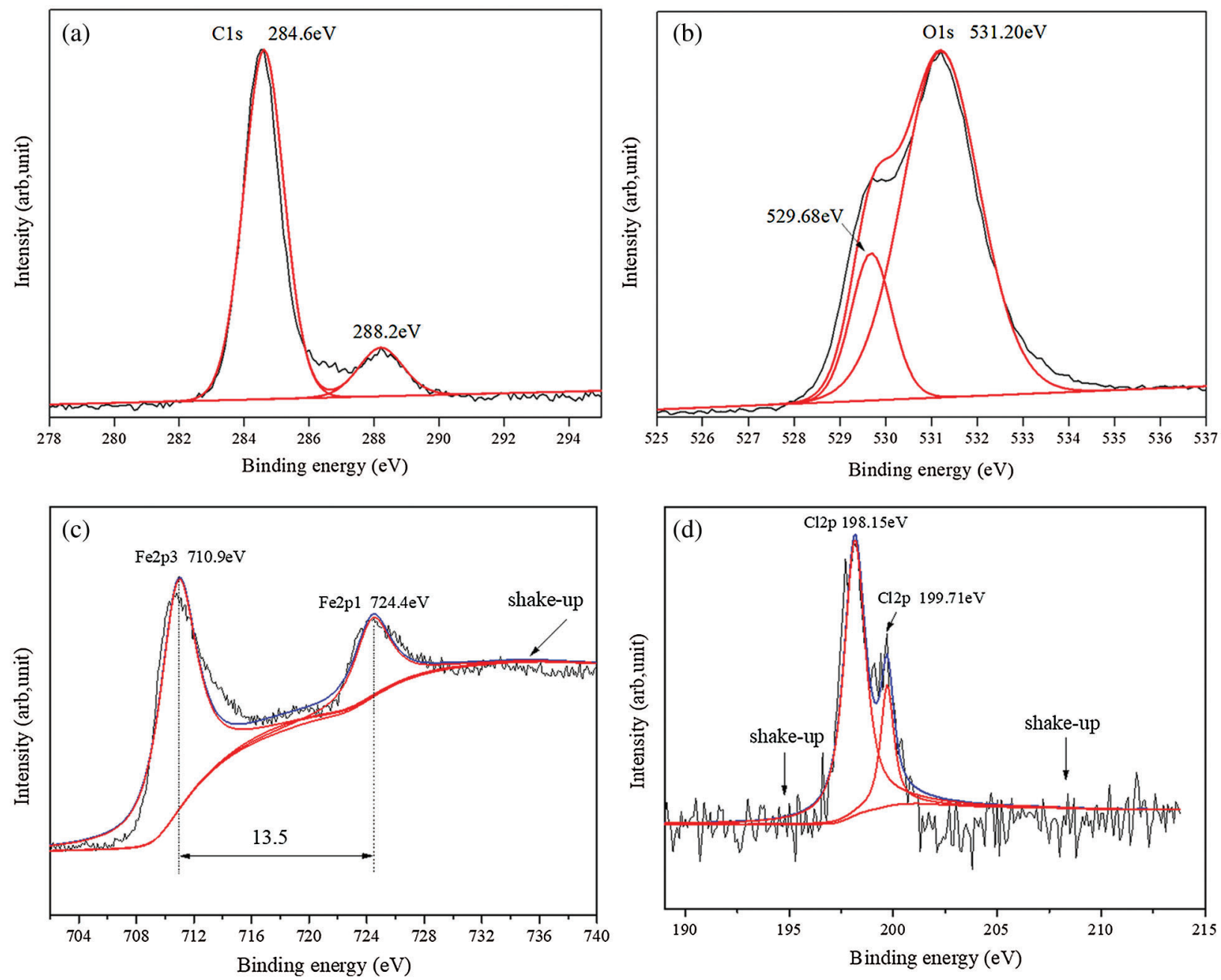

Figure 14: The (a) $\mathrm{C} 1 \mathrm{~s}$ peak, (b) $\mathrm{O} 1 \mathrm{~s}$ peak, (c) Fe2p peak, (d) Cl2p peak on $\mathrm{N} 80$ steel with $8 \mathrm{mmol} \cdot \mathrm{L}^{-1} \beta$-HA

\subsection{Quantum Calculations}

Based on the frontier molecular orbital theory, the adsorption centers of three kinds of organic corrosion inhibitors combined with carbon steel matrix through Lewis acid-base interaction were further revealed. The 
global minimum energy structure of the three inhibitors and the distribution of the highest occupied molecular orbital (HOMO) and the lowest unoccupied molecular orbital (LUMO) are shown in Fig. 15. The calculated parameters are listed in Tab. 7.
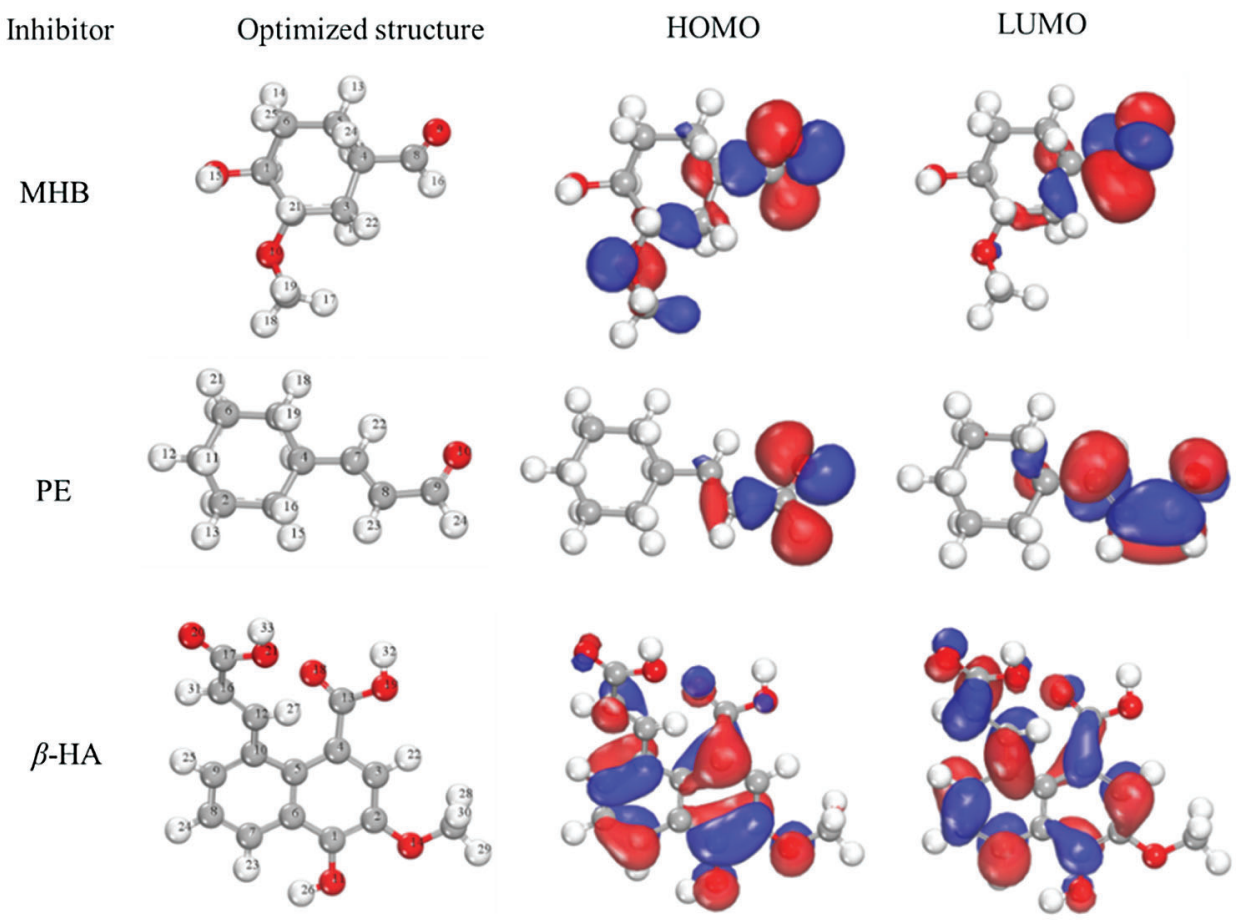

Figure 15: Optimized structure, HOMO and LUMO orbital distribution of MHB, PE and $\beta$-HA inhibitor molecules

Table 7: Quantum chemical parameters of MHB, PE and $\beta$-HA molecules

\begin{tabular}{lllllllll}
\hline Inhibitors & $\begin{array}{l}E_{\mathrm{HOMO}} \\
(\mathrm{eV})\end{array}$ & $\begin{array}{l}E_{\mathrm{LUMO}} \\
(\mathrm{eV})\end{array}$ & $\begin{array}{l}\Delta E \\
(\mathrm{eV})\end{array}$ & $\begin{array}{l}\chi \\
(\mathrm{eV})\end{array}$ & $\begin{array}{l}\eta \\
(\mathrm{eV})\end{array}$ & $\begin{array}{l}\sigma \\
\left(\mathrm{eV}^{-1}\right)\end{array}$ & $\begin{array}{l}\omega \\
(\mathrm{eV})\end{array}$ & $\begin{array}{l}\varepsilon \\
\left(\mathrm{eV}^{-1}\right)\end{array}$ \\
\hline MHB & -0.211 & -0.072 & 0.139 & 0.142 & 0.070 & 14.357 & 0.144 & 6.941 \\
PE & -0.204 & -0.096 & 0.108 & 0.150 & 0.054 & 18.571 & 0.209 & 4.787 \\
$\beta$-HA & -0.192 & -0.111 & 0.081 & 0.151 & 0.041 & 24.660 & 0.282 & 3.541 \\
\hline
\end{tabular}

The HOMO orbital can provide electrons to the vacancy metal orbital and interact with the metal atoms at the active site on the substrate surface, while the active site in the LUMO region of the inhibitor molecule can receive electrons from the filling orbital of the Fe atoms on the substrate surface [52]. For MHB and PE based on the distribution of HOMO and LUMO (Fig. 15), it can be seen that the HOMO orbit is concentrated on the $\mathrm{O}$ atoms and $\mathrm{C}$ atoms, while the LUMO orbital covers on the aldehyde group and its adjacent $\mathrm{C}$ atoms. For $\beta$-HA, the active centers of the HOMO orbital and the LUMO orbital were mainly located on $\mathrm{O}$ atoms, $\mathrm{C}$ atoms on benzene ring, and $-\mathrm{COOH}$. The results indicate that $\beta$-HA molecules can contribute delocalized $\pi$ electrons from benzene ring to the low energy vacancy orbit of $\mathrm{Fe}$ atoms on the surface, thus promoting the adsorption of inhibitor molecules. 
According to the results in Tab. 7, the $E_{\mathrm{HOMO}}$ of $\beta$-HA is higher than that of MHB and PE, which indicates that $\beta$-HA is easier to provide electrons to the unoccupied metal $\mathrm{d}$ orbit. Moreover, the $E_{\mathrm{LUMO}}$ of $\beta$-HA is lower than that of MHB and PE, illustrating that the ability to accept electron of $\beta$-HA is stronger than that of MHB and PE. Compared with MHB and PE, the energy gap $\triangle \mathrm{E}$ between HOMO and LUMO orbitals for $\beta$-HA is lowest, indicating inhibitor $\beta$-HA has better adsorption capacity on Fe (110) surface. Meanwhile, the $O$ heteroatom play an important role as the active center of the interaction between inhibitor and steel surface. The adsorption trends of three inhibitors were studied by using the Hard and Soft Acids and Bases (HSAB) theory [53]. As a soft acid, Fe atom is easier to coordinate with soft base. Based on the DFT calculation results, the interaction ability of $\beta$-HA molecule with metal surface is better than that of MHB and PE, which is in good agreement with the experimental data.

\subsection{Molecular Dynamic (MD) Simulation}

Fig. 16 shows the free volume distribution of 1000ps for three inhibitors, and the blue part is the free volume in the model space. Based on the simulation results, it can be seen that the free volume fraction (FFV) of $\beta$-HA inhibitor film is the lowest among the three kinds of inhibitor films, which indicates that the porosity of the $\beta$-HA film is the lowest. Meanwhile, the diffusion ability of corrosion medium molecules through the film to the substrate is the weakest after adding inhibitor $\beta$-HA into corrosion medium.

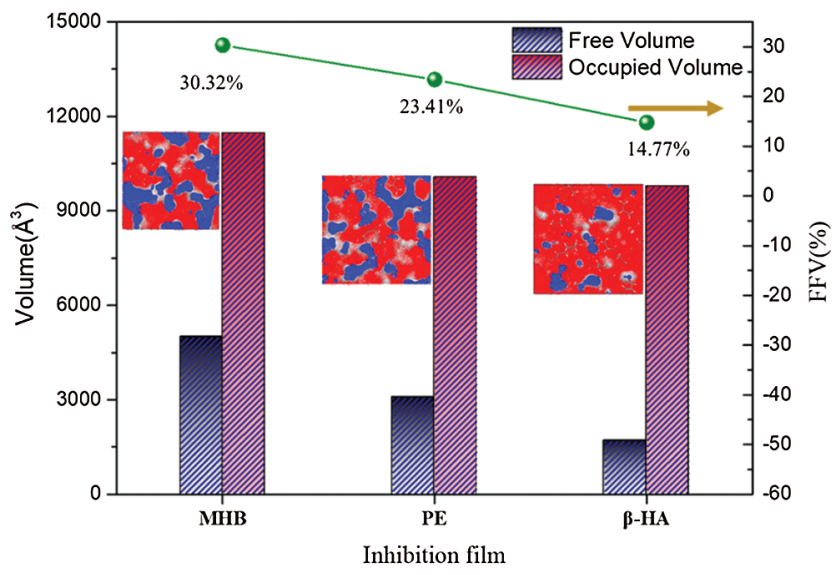

Figure 16: The distribution of free volume in simulated inhibitor layers

In order to better understand the adsorption of MHB, PE and $\beta$-HA on metal surface, the adsorption configurations of three inhibitors on the Fe (110) surface are shown in Fig. 17.

As shown in Fig. 17, all three inhibitor molecules are adsorbed on the Fe (110) surface in parallel. The parallel adsorption of the three inhibitors on the iron surface may be due to the homogeneous distribution of HOMO and LUMO orbital density. The inhibitor molecules cannot only form a coordination bond with the empty d-orbital on the iron surface by providing lone pair electrons, but also form an anti-bond by accepting electrons from the d-orbital with $\pi$ bond on the Fe surface.

By calculating the interaction energy $\left(E_{\text {binding }}\right)$ between the molecules and the Fe (110) surface, the adsorption level of inhibitor molecules with $\mathrm{Fe}$ matrix layer in simulated solvent was quantitatively studied. The binding energy of the three inhibitor molecules interacting with the Fe layer are shown in Tab. 8. The order of the interaction energies of the three inhibitor molecules with $\mathrm{Fe}(110)$ is $\beta$-HA $\left(-553.16 \mathrm{~kJ} \mathrm{~mol}^{-1}\right)<\operatorname{PE}\left(-329.94 \mathrm{~kJ} \mathrm{~mol}^{-1}\right)<\mathrm{MHB}\left(-312.69 \mathrm{~kJ} \mathrm{~mol}^{-1}\right)$, respectively. It can be seen

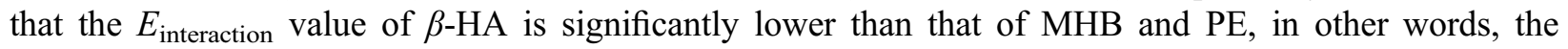
adsorption energy of $\beta$-HA is higher than that of MHB and PE. The higher adsorption energy suggests 
that the interaction between inhibitor molecules and the metal surface is relatively stable, and the inhibitor can show good inhibition performance.
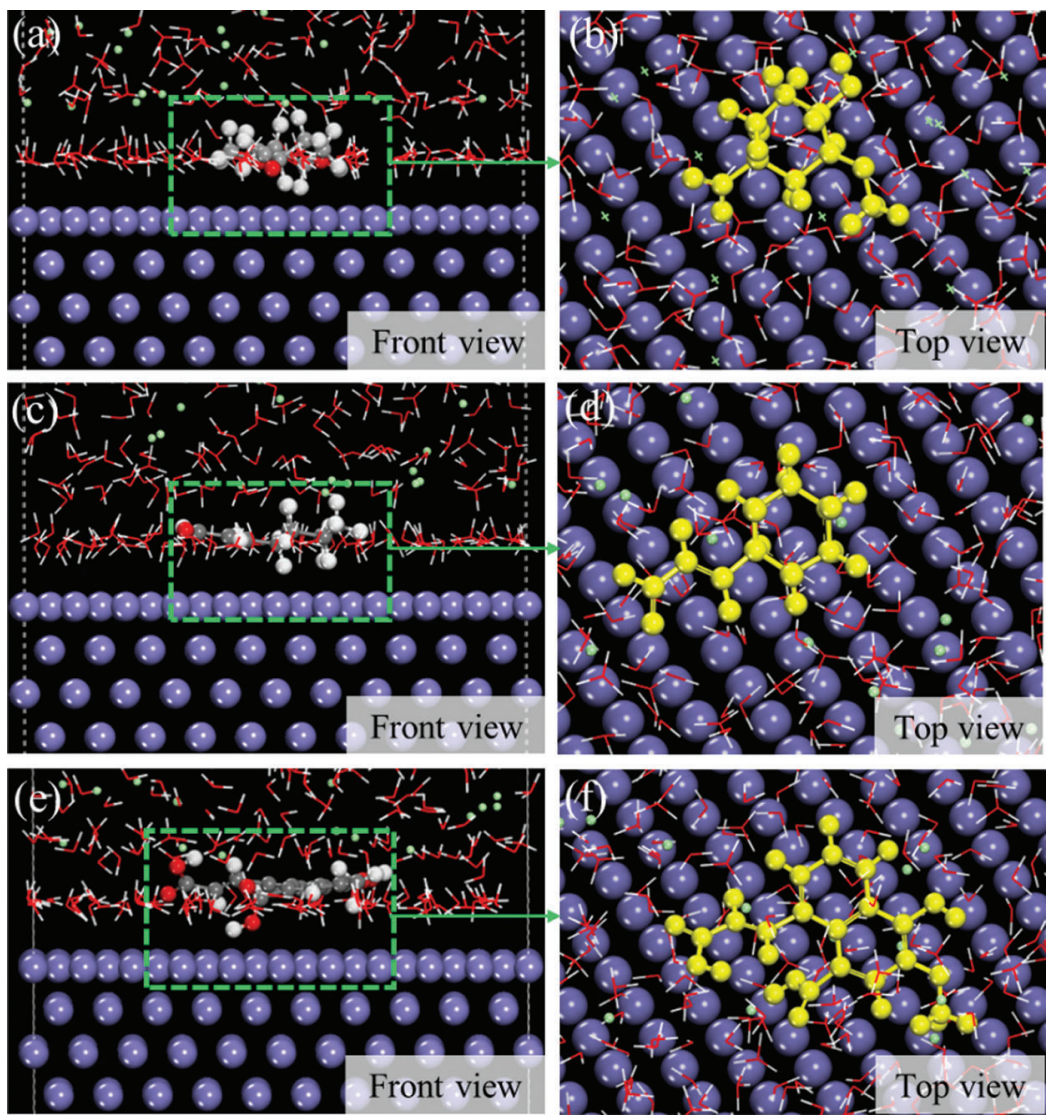

Figure 17: Adsorption configurations of MHB, $\mathrm{PE}$ and $\beta$-HA inhibitor molecules on $\mathrm{Fe}(110)$ surface in $\mathrm{HCl}$ solution, (a, d) MHB, (b and e) PE and (c and f) $\beta$-HA

Table 8: Binding energies of $\mathrm{MHB}, \mathrm{PE}$ and $\beta$-HA inhibitor molecules on the Fe (110) surface in the simulated $\mathrm{HCl}$ solution

\begin{tabular}{lllll}
\hline Inhibitors & Bond name & Length $\mathrm{nm}$ & $E_{\text {interaction }}\left(\mathrm{kJ} \mathrm{mol}^{-1}\right)$ & $E_{\text {binding }}\left(\mathrm{kJ} \mathrm{mol}^{-1}\right)$ \\
\hline MHB & Fe-O7 & 2.08 & -312.69 & 312.69 \\
PE & Fe-O10 & 1.96 & -329.94 & 329.94 \\
$\beta$-HA & Fe-O11 & 2.01 & -553.16 & 553.16 \\
& Fe-O14 & 1.99 & & \\
& Fe-O19 & 1.45 & & \\
& Fe-O21 & 1.69 & & \\
\hline
\end{tabular}

In order to verify the experimental results more accurately, the bond length of the interaction between the three inhibitors and the $\mathrm{Fe}(110)$ surface was calculated. Generally, if the bond length is less than $3.5 \AA$, there is a strong chemical bond between the heteroatom and the Fe atoms. On the contrary, the interaction force is 
van der Waals force [54]. According to the shortest bond distance between the $\mathrm{O}$ atom of three inhibitors and the surface of $\mathrm{Fe}(110)$ in Tab. 6, the results show that the three inhibitors are adsorbed by chemical bond on the surface of $\mathrm{Fe}(110)$. The order of the bond lowest distance is $\beta$-HA $(1.45 \mathrm{~nm})<\mathrm{PE}(1.96 \mathrm{~nm})<\mathrm{MHB}$ $(2.08 \mathrm{~nm})$. The adsorption of $\beta$-HA on Fe (110) surface is more stable, which can effectively block the migration of electrons at the interface of metal solution and the diffusion of chloride ions to metal surface. This suggests that the new $\beta$-HA has a good interface binding property and enhances the interaction between the inhibitor molecule and Fe surface.

\subsection{Inhibition Mechanism}

The corrosion of $\mathrm{N} 80$ steel in $\mathrm{HCl}$ solution belongs to electrochemical corrosion:

$\mathrm{Fe}-2 e^{-} \rightarrow \mathrm{Fe}^{2+}$

$H^{+}+2 e^{-} \rightarrow \mathrm{H}_{2} \uparrow$

It can be seen that the higher concentration of $\mathrm{H}^{+}$in hydrochloric acid medium accelerates the corrosion cathodic reaction of N80 steel, and further aggravates the dissolution of matrix. The surface of N80 samples in the $15 \% \mathrm{HCl}$ solution without corrosion inhibitor was irregular and occurred severe corrosion.

The corrosion inhibition of MHB, $\mathrm{PE}$ and $\beta$-HA is devoted to the $\mathrm{O}$ atoms with lone pair electrons in inhibitor molecules, which may interact with $\mathrm{Fe}$ atom by the coordination bond, neatly arranging on the N80 steel corrosion surface. According to the results of $\Delta G_{\text {ads }}$, the mixed adsorption process that involves both physical and chemical adsorption on the surface of N80 steel occurs. The ionization of acid radical ions in $\beta$-HA molecules and the electrostatic repulsion between molecular chains promote the spread of $\beta$-HA groups and form a film on the surface of N80 steel, which has a good inhibition effect on the corrosion of $\mathrm{N} 80$ steel in $\mathrm{HCl}$ medium. When the metal is in contact with acidic medium, the inhibitor can form a single molecule adsorption film on the metal surface, and the inhibition effect can be achieved by changing the redox potential of hydrogen ion. However, the inhibition efficiency of MHB and PE is lower, which is related to the physical adsorption of the film formed on the surface of N80 steel.

\section{Conclusion}

The MHB, PE and $\beta$-HA were chosen to be as corrosion inhibitor for $\mathrm{N} 80$ steel corrosion in $15 \% \mathrm{HCl}$ solution, and the corrosion inhibition performance was evaluated by using weight loss, electrochemical measurements, scanning electron microscope (SEM), quantum chemical calculation and molecular dynamics simulation (MDS).

(1) By compare the corrosion inhibition performance of three different organic, the synthesized $\beta$-HA inhibitor can provide better protection for N80 steel in hydrochloric acid solution. Corrosion inhibition efficiency has a trend of increase along the concentration. The adsorption of inhibitor molecules obeys Langmuir adsorption isotherm with the $\Delta G_{\text {ads }}$ at $-28.81 \mathrm{~kJ} \cdot \mathrm{mol}^{-1}$. In addition, the whole adsorption process can be divided into two stages: the first is that inhibitor molecules rapidly adsorbed on the N80 steel surface, and this is followed by large $\pi$ bond of benzene ring to combine with the empty orbital of Fe atoms to form more stable bonds. The formed film can successfully hinder chlorine contact with metal.

(2) Based on quantum chemical calculation and molecular dynamics simulation results, inhibitor $\beta$-HA has better adsorption capacity on $\mathrm{Fe}(110)$ surface and the Fe-O bong length of $\beta$-HA is lower than that of MHB and PE. Accordingly, the current work finds that inhibitor $\beta$-HA can greatly reduce the corrosion rate of N80 steel in hydrochloric acid medium. Since both MHB and PE are environmentally friendly chemicals, this finding is expected to possess a wider application in corrosion protection. 
Acknowledgement: The authors thank the postgraduate students their kind help. The authors also sincerely thank the anonymous reviewers for their constructive suggestions.

Funding Statement: This work was supported by the National Science and Technology Major Project of China (Grant No. 2016ZX05028-004) and the Fundamental Research Funds for the Central Universities (Grant No. FRF-IC-18-007).

Conflicts of Interest: The authors declare that they have no conflicts of interest to report regarding the present study.

\section{References}

1. Gu, C. Y., Di, Q. F., Wang, Z. H. (2006). Stress corrosion performance of N80 steel in formation water. Acta Physica Sinica, 27, 141-144. DOI 10.3321/j.issn:0253-2697.2006.02.031.

2. Yadav, M., Behera, D., Sharma, U. (2016). Nontoxic corrosion inhibitors for N80 steel in hydrochloric acid. Arabian Journal of Chemistry, 9, 1487-1495. DOI 10.1016/j.arabjc.2012.03.011.

3. Zhang, Q., Yang, W. Z., Tang, Y. M., Gu, C. Y., Zhou, S. Q. et al. (2006). Stress corrosion cracking of steel N80 in jiangsu oil field. Corrosion and Protection, 27, 66-68. DOI 10.3969/j.issn.1005-748X.2006.02.004.

4. Zhou, W. J., Yan, M. L., Wang, C. D. (2007). Corrosion behavior of N80 tubing steel in medium containing carbon dioxide hydrogen sulfide and high chlorine ions. Corrosion Engineering Science and Technology, 19, 192-195. DOI $10.1002 /$ jrs. 1570 .

5. Vishwanatham, S., Haldar, N. (2008). Furfuryl alcohol as corrosion inhibitor for N80 steel in hydrochloric acid. Corrosion Science, 50, 2999-3004. DOI 10.1016/j.corsci.2008.08.005.

6. Yadav, M., Sharma, U., Yadav, P. N. (2013). Isatin compounds as corrosion inhibitors for N80 steel in 15\%HCl. Egyptian Journal of Petroleum, 22, 335-344. DOI 10.1016/j.ejpe.2013.10.001.

7. Wang, X., Zhang, R. Y., Shangguan, C. H., Chen, Y. B. (2013). Corrosion behaviour of two casing steels in simulative oil field formation water containing $\mathrm{CO}_{2}$. Material Mechanics Engineering, 37, 69-72. DOI 10.1016/j.issn.1000-3738.2013.05.004.

8. Li, W. X., Li, R. L. (2005). Research and application of corrosion and protection technology in jidong oilfield. Journal of Oil \& Gas Technology, 964, 965-967. DOI 10.3969/j.issn.1006-0235.2017.11.084.

9. Ashassi, S. H., Majidi, M. R., Seyyedi, K. (2004). Investigation of inhibition effect of some amino acids steel corrosion in HCl solution. Applied Surface Science, 225, 176-185. DOI 10.1016/j.apsusc.2003.10.007.

10. Khaled, K. F. (2010). Corrosion control of copper in nitric acid solution using some aminoacids-a combined experimental and theoretical study. Corrosion Science, 52, 3225-3234. DOI 10.1016/j.corsci.2010.05.039.

11. Masoud, M. S., Awad, M. K., Shaker, M. A., EI-Tahawy, M. M. T. (2010). The role of structural chemistry in the inhibitive performance of some aminopyrimidines on the corrosion of steel. Corrosion Science, 52, 2387-2396. DOI 10.1016/j.corsci.2010.04.011.

12. Hu, S. Q., Guo, A. L., Yan, Y. G., Jia, X. L., Geng, Y. F. (2001). Computer simulation of diffusion of corrosive particle in corrosion inhibitor membrane. Computational and Theoretical Chemistry, 964, 176-181. DOI 10.1016/j.comptc.2010.12.019.

13. Mi, H., Xiao, G., Chen, X. (2015). Theoretical evaluation of corrosion inhibition performance of three antipyrine compounds. Computational and Theoretical Chemistry, 1072, 7-14. DOI 10.1016/j.comptc.2015.08.023.

14. Wang, Z. Z., Li, Y. Y., Zhang, G. A. (2019). Inhibitive effects of inhibitors on the galvanic corrosion between $\mathrm{N} 80$ carbon steel and $13 \mathrm{cr}$ stainless steel under dynamic supercritical $\mathrm{CO}_{2}$ conditions. Corrosion Science, 146, 121-133. DOI 10.1016/j.corsci.2018.10.028.

15. Singh, A., Ansari, K. R., Quraishi, M. A., Lgaz, H., Lin, Y. H. (2018). Synthesis and investigation of pyran derivatives as acidizing corrosion inhibitors for N80 steel in hydrochloric acid: Theoretical and experimental approaches. Journal of Alloys and Compounds, 762, 347-362. DOI 10.1016/j.jallcom.2018.05.236. 
16. Mourya, P., Banerjee, S., Singh, M. M. (2014). Corrosion inhibition of mild steel in acidic solution by tagetes erecta. (Marigold Flower) Extract as a Green Inhibitor. Corrosion Science, 85, 352-363. DOI 10.1016/j. corsci.2014.04.036.

17. Saraswat, V., Yadav, M. (2020). Computational and electrochemical analysis on quinoxalines as corrosion inhibitors for mild steel in acidic medium. Journal of Molecular Liquids, 297, 111883. DOI 10.1016/j. molliq.2019.111883.

18. Saraswata, V., Yadava, M., Obot, I. B. (2020). Investigations on eco-friendly corrosion inhibitors for mild steel in acid environment: Electrochemical: DFT and Monte Carlo Simulation approach. Colloids and Surfaces A: Physicochemical and Engineering Aspects, 599, 124881. DOI 10.1016/j.colsurfa.2020.124881.

19. Ma, Y. C., Fan, B. M., Hao, H., Lv, J. Y., Shang, C. S. et al. (2018). Assembly mechanism of a supramolecular complex based on trans-cinnamaldehyde on the mild steel in $0.5 \mathrm{M} \mathrm{HCl}$. Proceedings of 2018 National Symposium on Corrosion Electrochemistry and Testing Methods, pp. 15.

20. Su, T. J., Li, K. E. (2018). Inhibition behavior of N, N'-dicinnamylidene-o-phenylenediamine for mild steel in hydrochloric acid. Chemical Research and Application, 30, 62-68. DOI 10.3969/j.issn.1004-1656.2018.01.011.

21. El-Etre, A. Y. (2001). Inhibition of acid corrosion of aluminum using vanillin. Corrosion Science, 43, 1031-1039. DOI 10.1016/S0010-938X(00)00127-X.

22. Kumar, S. S., Priyadarsini, K. I., Sainis, K. B. (2004). Inhibition of peroxynitrite-mediated reactions by vanillin. Journal of Agricultural and Food Chemistry, 52, 139-145. DOI 10.1021/jf030319d.

23. Wang, Y., Hu, J., Wang, Y. Q., Yu, L. J. (2016). A new method for preventing corrosion failure: Thiourea and hexamethylenetetramine as inhibitor for copper. Bulletin of the Korean Chemical Society, 37, 1797-1811. DOI 10.1002/bkcs.10978.

24. Gupta, N. K., Verma, C., Quraishi, M. A., Mukherjee, A. K. (2016). Schiff's bases derived from l-lysine and aromatic aldehydes as green corrosion inhibitors for mild steel: Experimental and theoretical studies. Journal of Molecular Liquids, 215, 47-57. DOI 10.1016/j.molliq.2015.12.027.

25. Lin, X., Liu, C., Wang, J. B., Yang, S. (2019). Graphitic carbon nitride quantum dots and nitrogen-doped carbon quantum dots co-decorated with $\mathrm{BiVO}_{4}$ microspheres: A ternary heterostructure photocatalyst for water purification. Separation and Purification Technology, 226, 117-127. DOI 10.1016/j.seppur.2019.05.093.

26. Ansari, K. R., Quraishi, M. A., Singh, A. (2014). Schiff's base of pyridyl substituted triazoles as new and effective corrosion inhibitors for mild steel in hydrochloric acid solution. Corrosion Science, 79, 5-15. DOI 10.1016/j. corsci.2013.10.009.

27. Morgan, D. J. (2019). Imaging XPS for industrial applications. Journal of Electron Spectroscopy \& Related Phenomena, 231, 109-117. DOI 10.1016/j.elspec.2017.12.008.

28. Verma, C., Quraishi, M. A. (2017). 2-Amino-4-(2,4-dihydroxyphenyl) Quinoline-3-carbonitrile as sustainable corrosion inhibitor for SAE 1006 steel in $1 \mathrm{M} \mathrm{HCl}$ : Electrochemical and surface investigation. Journal of the Association of Arab Universities for Basic and Applied Sciences, 23, 29-36. DOI 10.1016/j.jaubas.2016.03.002.

29. Vinothkumar, K., Sethuraman, M. G. (2018). Corrosion inhibition ability of electropolymerised composite film of 2-amino-5-mercapto-1,3,4-thiadiazole/TiO2 deposited over the copper electrode in neutral medium. Materials Today Communications, 14, 27-39. DOI 10.1016/j.mtcomm.2017.12.007.

30. Hu, H., Li, X., Fang, Z., Wei, N., Li, Q. (2010). Small-molecule gas sorption and diffusion in coal: Molecular simulation. Energy, 35, 2939-2944. DOI 10.1016/j.energy.2010.03.028.

31. Yu, W. Z., Yan, Y. G., Yu, L. J., Ren, Z. J. (2010). Molecular dynamics simulation of the adsorption behavior of imidazoline corrosion inhibitors on a Fe [001] surface. Acta Physico-Chimica Sinica, 26, 1385-1390. DOI 10.3866/PKU.WHXB20100501.

32. Guo, L., Ye, G., Obot, I. B., Li, X., Shen, X. et al. (2017). Synergistic effect of potassium iodide with Ltryptophane on the corrosion inhibition of mild steel: A combined electrochemical and theoretical study. International Journal of Electrochemical Science, 12, 166-177. DOI 10.20964/2017.01.04.

33. Ye, Y. W., Yang, D. P., Chen, H., Guo, S. D., Yang, Q. M. et al. (2020). A high-efficiency corrosion inhibitor of Ndoped citric acid-based carbon dots for mild steel in hydrochloric acid environment. Journal of Hazardous Materials, 381, 121019. DOI 10.1016/j.jhazmat.2019.121019. 
34. El-Hajjaji, F., Ech-chihbi, E., Rezki, N., Benhiba, F., Taleb, M. et al. (2020). Electrochemical and theoretical insights on the adsorption and corrosion inhibition of novel pyridinium-derived ionic liquids for mild steel in $1 \mathrm{M} \mathrm{HCl}$. Journal of Molecular Liquids, 314, 113737. DOI 10.1016/j.molliq.2020.113737.

35. Yildiz, R. (2015). An electrochemical and theoretical evaluation of 4,6-diamino-2-pyrimidinethiol as corrosion inhibitor for mild steel in $\mathrm{HCl}$ solutions. Corrosion Science, 90, 544-553. DOI 10.1016/j.corsci.2014.10.047.

36. Singh, P., Quraishi, M. A. (2016). Corrosion inhibition of mild steel using novel bis schiff's bases as corrosion inhibitors: Electrochemical and surface measurement. Measurment, 86, 114-124. DOI 10.1016/j. measurement.2016.02.052.

37. Paul, P. K., Yadav, M. (2020). Investigation on corrosion inhibition and adsorption mechanism of triazine-thiourea derivatives at mild steel/HCl solution interface: Electrochemical, XPS, DFT and monte carlo simulation approach. Journal of Electroanalytical Chemistry, 877, 114599. DOI 10.1016/j.jelechem.2020.114599.

38. Ayagou, M. D. D., Tran, T. T. M., Tribollet, B., Kittel, J., Sutter, E. et al. (2018). Electrochemical impedance spectroscopy of iron corrosion in $\mathrm{H}_{2} \mathrm{~S}$ solutions. Electrochimica Acta, 282, 775-783. DOI 10.1016/j. electacta.2018.06.052.

39. Emori, W., Zhang, R. H., Okafor, P. C., Zheng, X. W., Cheng, C. R. (2020). Adsorption and corrosion inhibition performance of multi-phytoconstituents from dioscorea septemloba on carbon steel in acidic media: Characterization, experimental and theoretical studies. Colloid Surfaces A, 590, 124534. DOI 10.1016/j. colsurfa.2020.124534.

40. Musa, A. Y., Kadhum, A. A. H., Mohamad, A. B., Takriff, M. S., Daud, A. R. et al. (2010). Adsorption isotherm mechanism of amino organic compounds as mild steel corrosion inhibitors by electrochemical measurement method. Journal of Central South University Technology, 17, 34-39. DOI 10.1007/s11771-010-0007-5.

41. Ye, Y., Zhang, D., Li, J., Liu, T., Pu, J. et al. (2019). One-step synthesis of superhydrophobic polyhedral oligomeric silsesquioxane-graphene oxide and its application in anti-corrosion and anti-wear fields. Corrosion Science, 147, 9-21. DOI 10.1016/j.corsci.2018.10.034.

42. Faydy, M. E., Touir, R., Touhami, M. E., Zarrouk, A., Jama, C. et al. (2018). Corrosion inhibition performance of newly synthesized 5-alkoxymethyl-8-hydroxyquinoline derivatives for carbon steel in $1 \mathrm{M} \mathrm{HCl}$ solution: Experimental, DFT and monte carlo simulation studies. Physical Chemistry Chemical Physics, 20, 20167. DOI 10.1039/C8CP03226B.

43. Zhao, Q., Guo, J. X., Cui, G. D., Han, T., Wu, Y. H. (2020). Chitosan derivatives as green corrosion inhibitors for p110 steel in a carbon dioxide environment. Colloid Surface B, 194, 111150. DOI 10.1016/j.colsurfb.2020.111150.

44. Zhang, J., Zhang, L., Tao, G. (2018). A novel and high-efficiency inhibitor of 5-(4-methoxyphenyl)-3 h-1,2dithiole-3-thione for copper corrosion inhibition in sulfuric acid at different temperatures. Journal of Molecular Liquids, 272, 369. DOI 10.1016/j.molliq.2018.09.095.

45. Machnikova, E., Whitmire, K. H., Hackerman, N. (2008). Corrosion inhibition of carbon steel in hydrochloric acid by furan derivatives. Electrochimica Acta, 53, 6024-6032. DOI 10.1016/j.electacta.2005.11.002.

46. Wan, W., Luo, J., Huang, C., Yang, J., Feng, Y. B. et al. (2018). Calcium copper titanate/polyurethane composite films with high dielectric constant, low dielectric loss and super flexibility. Ceramics International, 44, 50865092. DOI 10.1016/j.ceramint.2017.12.108.

47. Pasquino, R., Panariello, D., Grizzut, N. (2013). Migration and alignment of spherical particles in sheared viscoelastic suspensions. a quantitative determination of the flow-induced self-assembly kinetics. Journal of Colloid and Interface Science, 394, 49-54. DOI 10.1016/j.jcis.2012.11.035.

48. Ye, Y. W., Yang, D. P., Chen, H. (2019). A green and effective corrosion inhibitor of functionalized carbon dots. Journal of Materials Science \& Technology, 35, 2243-2253. DOI 10.1016/j.jmst.2019.05.045.

49. Hu, J., Wang, Y., Yu, L. J., Zheng, M. S., Zhao, Y. (2014). Electrochemical analysis of inhibition by silver sulfate for alloy690 in chloride solution. CIESC Journal, 10, 4032-4038. DOI 10.3969/j.issn.0438-1157.2014.10.037.

50. Nakayama, N., Obuchi, A. (2003). Inhibitory effects of 5-aminouracil on cathodic reactions of steels in saturated $\mathrm{Ca}(\mathrm{OH})_{2}$, solutions. Corrosion Science, 45, 2075-2092. DOI 10.1016/s0010-938x. 
JRM, 2022, vol.10, no.2

51. Pech-Canul, M. A., Bartolo-Pérez, P. (2004). Inhibition effects of N-phosphono-methyl-glycine/ $\mathrm{Zn}^{2+}$, mixtures on corrosion of steel in neutral chloride solutions. Surface and Coatings Technology, 184, 133-140. DOI 10.1016/j. surfcoat.2003.11.018.

52. Zhang, Z., Li, W. W., Zhang, W. P., Huang, X. D., Ruan, L. et al. (2018). Experimental, quantum chemical calculations and molecular dynamics (MD) simulation studies of methionine and valine as corrosion inhibitors on carbon steel in phase change materials (PCMs) solution. Journal of Molecular Liquids, 272, 528-538. DOI 10.1016/j.molliq.2018.09.081.

53. Wang, Y., Wang, Z., Cao, J. L., Lu, M. X., Zhang, L. (2020). Theoretical insights into the inhibition performance of three neonicotine derivatives as novel type of inhibitors on carbon steel. Journal of Renewable Materials, 8, 819832. DOI 10.32604/jrm.2020.09395.

54. Saha, S. K., Banerjee, P. (2015). A theoretical approach to understand the inhibition mechanism of steel corrosion with two aminobenzonitrile inhibitors. RSC Advance, 5, 71120-71130. DOI 10.1039/C5RA15173B. 